\title{
Entretien avec Aline Schulman (MEDET LAT France 1945-2000)
}

\author{
Gustavo Guerrero, Bénédicte VAUthIER \& Roland BÉHAR \\ (Transcripción: Diana Teixera Coelho \& Bénédicte Vauthier) \\ Université Cergy-Pontoise, Universität Bern \& École Normale Supérieure
}

\begin{abstract}
El I5 de febrero de 2019 Gustavo Guerrero, Bénédicte Vauthier y Roland Béhar entrevistaron en París a Aline Schulman, traductora al francés de tres figuras emblemáticas de la literatura latinoamericana : el chileno José Donoso y los cubanos Severo Sarduy y Reinaldo Arenas y durante años traductora fiel de Juan Goytisolo. A finales de los años noventa, Aline Schulman ofrece una nueva traducción del Quijote, aclamada por los lectores. Le seguirá la traducción de otros clásicos de la literatura de los siglos de Oro: La Celestina, el Buscón y santa Teresa. Se ofrece aquí una transcripción íntegra de la entrevista (de unas dos horas). La traductora vuelve sobre la génesis, los motivos, las dificultades de su trabajo.
\end{abstract}

Keywords: Aline Schulman, traduction, traductrice, Donoso, Sarduy, Arenas, Juan Goytisolo, Don Quijote, Buscón, La Celestina, santa Teresa.

\section{Introducción}

La entrevista con la traductora Aline Schulman (AS), que se transcribe de forma íntegra a continuación, tuvo lugar el I5 de febrero de 2019 en la Escuela Normal Superior de París. Participaron en ella Bénédicte Vauthier (BV, Universidad de Berna), especialista de Juan Goytisolo, Roland Béhar (RB, École Normale Supérieure), especialista de la literatura del Siglo de Oro y Gustavo Guerrero (GG, Université Cergy-Pontoise Paris), promotor del acto, especialista de literaturas y culturas latinoamericanas, en particular de Severo Sarduy.

La entrevista ${ }^{2}$ se enmarca en las actividades de MEDET LAT ${ }^{3}$ cuyo objetivo es desarrollar y ofrecer una primera perspectiva de conjunto sobre

I Diana Teixeira Coelho, estudiante de Master en Lengua y Literaturas Hispánicas y Lengua y Literaturas Francesas de la Universidad de Berna, se hizo cargo de la transcripción íntegra de las dos horas de entrevista. Bénédicte Vauthier la revisó, haciendo leves reajustes, generalmente de carácter sintáctico, para adaptar la conversación al formato de una comunicación "oralizada" escrita. Con vistas a que se pueda o bien escuchar, o bien leer (si acaso citar) la entrevista, en ningún momento se intentó reescribirla, lo que explica, por ejemplo, que se hayan mantenido las repeticiones, etc.

2 De próxima escucha en el portal de Medet Lat: https://www.projet-medetlat.com

3 MEDET LAT (Mediación editorial, difusión y traducción de la literatura latinoamericana en Francia, 1945-2000) es un proyecto interdisciplinario elaborado por un grupo de hispanistas franceses y que se sitúa en el punto de encuentro entre los estudios literarios, la traduc- 
el papel de los principales actores franceses y latinoamericanos (escritores, agentes, editores, traductores, críticos) en este capítulo de la historia de la literatura mundial mediante el análisis de la producción y recepción de obras latinoamericanas traducidas entre 1945 y 2000. Se parte del examen de la documentación presente en archivos públicos y privados franceses, como los del Institut Mémoires de l'Édition Contemporaine (IMEC) o la Bibliothèque Nationale de France, pero también se ha diseñado un importante programa de entrevistas con los principales traductores, críticos y editores.

Este programa ocupa un lugar especial en el proyecto MEDET LAT. Preparadas de antemano, a través de varias reuniones previas entre el entrevistado/ la entrevistada y sus entrevistadorXs, las entrevistas permiten analizar la carrera de diferentes mediadorXs y ofrecen una mejor comprensión de los contextos históricos y del papel que estos desempeñaron en el proceso de circulación de obras latinoamericanas en Francia. Las entrevistas son además la ocasión de invitar a los principales especialistas de un autor o una obra traducida a fin de compartir sus trabajos y de atraer nuevas miradas hacia la realización del proyecto.

\section{Les débuts du travail de traductrice d'Aline Schulman, sa rencontre avec les littératures de l'Amérique latine : traduire Donoso, Sarduy et Arenas (Gustavo Guerrero)}

GG : Merci d'avoir accepté notre invitation, Aline Schulman. Vous êtes née à Casablanca et vous avez grandi dans cette ville. Ensuite, vous avez fait vos études en France et vous êtes devenue d'abord professeure à l'Université de Rennes puis à la Sorbonne, où vous avez enseigné la littérature espagnole et latino-américaine contemporaine jusqu'en 1999.

Très tôt vous vous êtes orientée vers la traduction. Vous publiez chez Gallimard, en 1969, une anthologie du poète Luis Cernuda : La realidad y el deseo. Juan Goytisolo, qui était à l'époque le conseiller littéraire pour l'Espagne et l'Amérique latine de la maison Gallimard, vous propose ensuite de traduire son roman Don Julian qui paraît en 1971 et à partir de là vous allez devenir sa traductrice attitrée avec pas moins de douze livres de Juan Goytisolo traduits en français.

Dans les années 1960-1970, vous vous intéressez aussi à la littérature latino-américaine, qui connaît alors un boom planétaire. On l'appelle le boom de la littérature latino-américaine. Vous traduisez le Chilien José

ción, la historia de la edición y la lectura, y las humanidades digitales. Su objetivo es analizar, desde un punto de vista histórico e intercultural, la participación de lXs editorXs, IXs traductorXs y lXs críticXs franceses en el proceso de circulación y reconocimiento internacional de las literaturas de América latina durante la segunda mitad del siglo xx. 
Donoso, notamment le roman Ce lieu sans limites, publié par Calmann-Lévy en 1973, puis les Cubains Reinaldo Arenas et Severo Sarduy, et plus tard les nouvelles de l'écrivain mexicain Carlos Fuentes.

À partir des années 1990, vous vous lancez dans la traduction de grands textes littéraires du Siècle d'Or. Rien de moins, vous vous lancez dans une traduction de Don Quichotte qui apparaît au Seuil en I997, puis de La Célestine, ensuite du roman picaresque de Quevedo, El Buscón, et plus récemment vous avez traduit une Anthologie des textes de Santa Teresa de Ávila, Le chemin de la perfection, publiée chez Fayard.

Merci et bienvenue à ce séminaire MEDET LAT, Aline Schulman.

AS : Merci, Gustavo, de m'accueillir ici surtout. Je suis très émue. Ça n'a peut-être pas l'air, mais c'est vrai, d'être à l'École Normale Supérieure et de venir parler d'une carrière, parce qu'en fait c'est toute une vie [...] dont il va falloir que, à travers vous enfin, je me justifie, il va falloir que je me justifie de ma vie entière. Ça ne va pas être facile.

GG : Pas du tout. Mais, on va commencer effectivement chronologiquement avec une question sur vos années de formation. Je viens de le dire, vous êtes née à Casablanca au sein d'une famille polyglotte et habituée à la coprésence de plusieurs langues dans son quotidien. Je voudrais savoir si vous avez un souvenir précis de votre apprentissage de l'espagnol. À quel moment s'est produite votre rencontre avec la langue espagnole et à quel moment vous décidez, vous voyez que votre vocation est celle de devenir une hispaniste?

AS : Ce serait tellement long à raconter que ça va être dit en trois phrases, autrement ça n'est pas possible. [...] Pour des raisons historiques, ma famille a voyagé en Argentine. Nous sommes arrivés à Buenos Aires le jour de mes quatre ans, voilà. Je n'irai pas dire quelle date, quelle année, non. On devine ou pas; et puis, nous en sommes repartis quand j'en avais cinq et demi, c'est-à-dire que j'ai appris à lire et à écrire en espagnol. Ensuite, retour au Maroc d'où nous arrivions où on parlait l'espagnol et le français, donc l'espagnol n'a jamais quitté véritablement ma vie. Je lisais Cinderella en anglais parce que ma mère était Anglaise, je lisais Cendrillon parce que j'étais Française et je lisais La Cenicienta parce que je parlais espagnol. Donc, dès le départ, il y a eu trois langues chez moi, dont l'espagnol, je ne dirais pas en priorité, mais à égalité avec le français. À partir de là, je dois vous dire que ce qui m'a encouragée à faire l'espagnol, c'était un professeur que j'avais en seconde, qui s'appelait Madame Hoyo, qui devait certainement être d'origine espagnole, qui nous a expliqué, ou dit, San Juan de la Cruz " como nadie ", comme personne. Et la Noche oscura m'est restée à vie, gravée, et je me suis dit «ça ce serait formidable ». Entretemps, la vie aidant, je voulais faire médecine et je me suis dit l'espagnol «bof ». Nous arrivons en France (puisque l'indépendance du Maroc etcetera), nous arrivons à Paris. Je 
finis mes études à Paris et puis je tombe amoureuse, comme tout le monde aussi, et mon amoureux me dit : « si tu fais médecine on ne se verra plus, fais espagnol ». Et c'est de là que tout a découlé. Alors, quand on dit vocation, c'est très vite dit, on peut toujours faire semblant, mais en vérité c'était ça. Donc, j'ai fait espagnol et je suis entrée à l'Institut Hispanique que ma petite fille appelait l'institut "panique ». C'était pas mal. Voilà pour vous donner une petite idée. C'était ça, la question que vous m'avez posée. Donc, il y a eu cette découverte de l'Espagne à travers cette professeure de seconde. Et il n'y avait pas seulement San Juan, mais il y avait Unamuno, il y avait bien sûr Lorca, il y avait tout ce monde merveilleux qu'elle m'a fait découvrir.

GG : Votre sensibilité littéraire a été formée par la lecture de ces classiques?

AS : Voilà. Et puis ma sensibilité littéraire, il faut aussi que je dise que mon amoureux était un (je ne donnerai pas son nom, je vais l'appeler Chano) très grand compositeur contemporain, de musique, classique bien sûr, et c'est à travers lui que j'ai vraiment connu Lorca avec le Llanto por Ignacio Sánchez Mejías. On parlait espagnol ensemble, il me lisait sans arrêt des poèmes de San Juan et de Lorca. Il me disait même cette phrase, je vous dirai entre guillemets, « après l'amour ", j'avais faim, bon, alors il me donnait du chocolat, et il me disait, en se trompant d'ailleurs, comme je m'en suis rendue compte après coup : "Come Sancho que yo nací para vivir queriendo, $y$ tú para morir comiendo ». Or, quand j'ai traduit Le Quichotte, tout d'un coup je tombe sur cette phrase et la vraie phrase, c'était quoi ? "Come Sancho que yo nací para morir queriendo y tú para vivir comiendo ». Alors, je suis très contente d'avoir pu rétablir la vérité.

GG : Ça a commencé par une mauvaise traduction, alors.

AS : Exactement. Exactement.

GG : Je suis très intéressé par votre rapport avec l'espagnol parce qu'il commence par la littérature, par l'amour de la littérature, finalement.

AS : Par l'amour de la littérature, par, comment dirais-je, par une nécessité vitale, je dirais d'abord. Le fait qu'on parle avec les amis, on parle et puis il y a la radio, vous savez Radio Tanger y Radio Andorra. Qu'est-ce qu'on entend au Maroc ? Alors, "para Pepita de dolorcita con no sé qué no sé cuánto ». On est sans arrêt dans la musique aussi. Donc, il y a à la fois la littérature, il y a la musique, et puis il y a le quotidien. C'est un ensemble, la langue espagnole pour moi.

GG : Vos premières traductions, nous avons parlé de la traduction de $\mathrm{La}$ réalité et le désir, La realidad y el deseo de Cernuda, c'étaient vos premières traductions? Vous avez commencé par Cernuda ou il y a eu d'autres essais préalables?

AS : Il y a eu même pas un essai. Vous savez la poésie pour moi, c'était l'espagnol, comme je vous ai dit, c'était Lorca, c'était San Juan. Or, je me 
trouve tout d'un coup, j'avais dix-huit ans, face à une poétesse française dont tout le monde ignore le nom ici, Sabine Sicaud. Mais, si vous regardez sur Internet, vous le trouvez. Et je me suis dit, ce n'est pas possible. Ça, ça doit être mis en espagnol, parce que c'est trop beau. Et j'ai traduit une dizaine de poèmes de Sabine Sicaud qui ont été publiés dans les Cuadernos Hispanoamericanos. Donc, à dix-neuf ans, j'avais fait le chemin inverse de traduire du français en espagnol. Donc, Cernuda, c'était ma deuxième traduction poétique, mais cette fois de l'espagnol en français.

GG : C'est Juan Goytisolo qui vous a demandé de traduire Cernuda?

AS : Pas tout à fait. C'est Monsieur Robert Marrast, notre regretté [...] professeur hispaniste, que j'ai toujours beaucoup aimé, et qui m'a toujours beaucoup aidée et qui, sachant que je faisais une thèse sur Cernuda, et à qui Juan Goytisolo avait demandé la traduction d'une anthologie de Cernuda, m'a dit : «Aline, vous ne voudriez pas m'aider?, parce que j'ai beaucoup de travail ». Et c'est comme ça que ça a commencé.

GG : D'accord, donc c'est par l'université que vous arrivez...

AS : C'est par l'université, par l'université.

GG : Ensuite, c'est Juan Goytisolo qui vous demande de traduire Don Julian en I969, le roman paraît en I97I, mais nous sommes à Paris et c'est le boom de la littérature latino-américaine. Vous êtes professeure à Rennes à l'époque et vous rencontrez Claude [...] Fell et Albert Bensoussan. [...] Les deux sont à Rennes. Est-ce que l'influence de l'un et de l'autre a été déterminante pour vos choix d'auteurs latino-américains, pour votre découverte de l'Amérique latine?

AS : Ma découverte de l'Amérique latine peut-être pas. J'étais surtout très liée avec Albert Bensoussan et Albert Bensoussan était lui-même très bien introduit chez Calmann-Lévy. Il traduisait déjà et donc c'est lui qui m'a proposé de traduire El lugar sin límites. Alors, moi je n'allais pas dire non, alors j'ai dit oui. Mal m'en a pris, car ce que je ne vous ai pas dit, c'est que si avec Juan la chose s'est passée au mieux, mais je crois qu'on parlera de Juan une autre fois, c'est parce que nous avions des liens communs. Il habitait, il avait écrit Don Julian à Tanger et moi j'avais habité à Tanger pendant trois ans. Donc, nous avions des, comment dirais-je, des points de repère géographiques, comment dirais-je, identiques. Or, le Chili, le Chili du bout du monde de El lugar sin limites, pour moi, c'était un lieu totalement inconnu. Et j'ai eu un mal fou à le traduire. Vraiment beaucoup beaucoup de mal, parce que je ne sais pas si vous serez d'accord avez moi et si les traducteurs seront d'accord avec moi, mais pour traduire il faut visualiser, c'est-à-dire, il faut d'une certaine manière reconnaître, non pas connaître, mais reconnaître de quoi il est question. Or, moi si je ne visualise pas, je suis incapable de traduire. Or, ce bout du monde chilien, je ne le voyais absolument pas. Et je 
peux avouer que si Albert Bensoussan n'avait pas été là pour reprendre ma traduction, c'était une vraie catastrophe.

GG : Je vais vous citer en 1987, lors de la table ronde aux Assises de la Traduction à Arles, je vous cite, vous dites ceci : « Le traducteur doit être capable d'apprécier le niveau exact de familiarité ou de vulgarité d'une expression, ne pas faire parler un camionneur du fin fond du Chili à la manière d'un grand chambellan, comme cela m'est arrivé à mes débuts ».

AS : Absolument et c'est de ce livre dont je voulais parler, mais je ne veux plus en parler parce que voilà, je crois que j'ai dit tout ce que j'avais à dire sur ce livre.

GG : Votre relation avec Bensoussan est très importante à cette époque, pour le début de votre carrière de traductrice, c'est lui qui vous introduit chez Calmann-Lévy?

AS : Oui.

GG: C'est lui qui vous relit aussi vos premières traductions de l'espagnol?

AS : Ben, c'est-à-dire pas mes premières. Si vous voulez, mon introduction c'est chez Gallimard, mais entre Don Julian et Juan sin tierra, Albert me demande de traduire et à ce moment-là je le fais, parce que je me suis dit, ça y est, je suis traductrice, j'y vais. Tu parles. Je ne m'attendais pas à ça du tout et donc, je ne suis pas traductrice encore puisque je suis incapable de traduire n'importe quoi. Et donc Albert m'a aidée ; il a relu et réécrit les passages qui étaient mauvais et après je suis retournée avec délices avec Juan, parce qu'avec Juan il y avait justement ce rapport, si vous voulez, de reconnaissance, de reconnaissance visuelle que j'avais avec lui et ses livres.

GG : Est-ce que vous avez fait appel à José Donoso pour résoudre les problèmes posés par la traduction de son roman ? Est-ce que vous avez eu du contact avec lui ? Est-ce que vous avez échangé avec lui à propos de la traduction de Ce lieu sans limites?

AS : Alors, malheureusement c'est un des seuls auteurs que j'aie jamais traduits, un des seuls, je ne dis pas le seul, avec lequel je n'ai jamais eu de contact. Je savais qu'il existait, mais je ne savais pas où et quand je ne savais pas, heureusement Albert était là. Alors, c'est Albert qui a été mon Donoso français, si vous voulez. C'est lui qui m'a aidée. Voilà, non, je n'ai jamais eu de contact avec Donoso.

GG : Et par la suite, vous ne l'avez jamais rencontré non plus?

AS : Jamais, jamais.

GG : Et vous n'avez jamais eu le désir ou l'intention de reprendre ce premier roman?

AS : De reprendre l'auteur ou de reprendre?

$\mathrm{GG}$ : De reprendre la traduction.

AS : Mais Albert l'avait tellement bien fait. Je n'ai aucune raison de le faire. Ça ne peut pas être mieux que ce qu'il fait, vous comprenez. 
GG : Très bien. Et plus tard, dans les années I990, vous devenez la traductrice de grands écrivains cubains, de Severo Sarduy et de Reinaldo Arenas. Lorsqu'on lit la correspondance de Reinaldo Arenas, on découvre qu'il n'y pas eu seulement une grande rivalité entre Sarduy et Arenas, mais que vous avez été l'un des motifs de cette rivalité entre Arenas et Sarduy. Dans ses lettres, Arenas accusait ou accuse Sarduy de monopoliser votre travail pour l'écarter justement de ses romans à lui. C'est-à-dire, comme si Severo Sarduy avait fait le nécessaire pour que vous ne traduisiez pas vraiment l'œuvre d'Arenas. Comment avez-vous géré cette rivalité entre les deux? Cette rivalité a-t-elle eu une quelconque influence sur le choix des œuvres que vous avez traduites de l'un et de l'autre?

AS : Vous savez, il faut vous dire que les grands, c'étaient Sarduy et Donoso. Moi je regardais, je comptais les coups, si vous voulez, mais je n'avais pas vraiment mon mot à dire. Severo me disait : «Tú conmigo. Ya está ». Et comme Donoso [sic] n'était pas là, il était le grand perdant. Severo était l'homme présent, c'était l'homme qui au Seuil faisait ce qu'il avait à faire. Alors voilà Severo me disait : «Tu me traduis ça, s’il te plaît ", je ne pouvais pas dire non. [...] Voilà, c'est, si vous voulez, il y a un rapport de force qui s'établit, je dirais, naturellement, entre ceux qui sont présents et ceux qui sont absents.

GG : C'était le début de votre collaboration avec le Seuil ou vous aviez déjà travaillé avec la maison de la Rue Jacob?

AS : Je crois que c'étaient mes débuts de collaboration avec le Seuil, oui, puisque jusqu'alors c'était chez Gallimard que je traduisais Juan. Oui, oui, oui c'étaient les débuts avec Severo. Alors, Severo qui me recevait dans son bureau, enfin, c'était le grand seigneur quoi. Voilà.

GG : Et en dehors de Sarduy, vous avez eu d'autres traductions au Seuil à l'époque, ou c'est seulement Sarduy...?

AS : J'en ai eu un, que Sarduy avait choisi, qui s'appelait La fase del rubí et que j'avais traduit par Le vitrail écarlate, qui est une dame (je vous dis tout de suite que j'ai oublié le nom à l'instant), qui n'a rien..., enfin ça n'a pas donné de petits, si je peux dire, voilà. Et à part ça, une fois que Severo a été traduit, c'est Juan qui a immigré, si je puis dire, au Seuil, donc on a continué à traduire Juan.

GG : D'accord. Dans le cas de Sarduy, les deux livres que vous avez traduits de son vivant, Colibrí et Pour que personne ne sache que j'ai peur, qui s'intitule Cocuyo [...] en espagnol, tous les deux portent la mention en français « traduit de l'espagnol par Aline Schulman et l'auteur ». Il y a eu donc un travail de collaboration entre vous et il s'agit d'un travail de collaboration qui, quand on le regarde de près, on découvre qu'il va bien au-delà de ce qu'on peut imaginer, car [...] on découvre, par exemple, que le roman a été restructuré au cours de la traduction. Il y a des chapitres qui ne sont pas 
dans l'édition espagnole et qui apparaissent dans l'édition française, il y a des paragraphes qui disparaissent, la structure du livre passe de trois à quatre parties. Donc, on a l'impression que vous et Sarduy vous vous êtes lancés dans une réécriture du roman au cours de la traduction, en particulier de Colibrí, comme si la version espagnole était un brouillon de roman qu'il fallait affiner ou perfectionner en français. Et j'aimerais bien que vous reveniez sur cette manière de travailler avec Sarduy et sur ce travail de traduction comme réécriture en fait que vous avez réalisé avec lui.

AS : Alors, je commence par la fin de ce que vous venez de dire, c'est-àdire, le brouillon. Vous savez que Borges dit que toutes les traductions ne sont que des brouillons, et que l'original est lui-même un brouillon possible. [...] Ça c'est une chose, mais ce qui est important, c'est qu'avec Sarduy et puis aussi avec Juan, mais j'en parlerai plus tard, comment vous dire, les textes étaient hyper baroques. Nous savons comment Sarduy traduisait. Il était influencé non seulement par son origine, mais par Tel Quel. Il était, il faisait partie de la troupe, si je puis dire, de Tel Quel, de Sollers, etcetera et il avait besoin d'être dans le baroque extrême. J'arrive, moi, avec ma traduction, nous sommes face à face et puis je lui dis ça, je ne peux pas le dire. Mais, il dit «bon, cambiar, on change ». On change, mais ce n'est pas moi qui change, c'est lui qui change, voyez-vous, et alors ce qu'il y avait d'extraordinaire c'est que je me suis rendu compte qu'au fond tout ce qu'il voulait, c'est que ça soit lu, lisible en français, parce que quand on traduit, on traduit pour être lu.

GG : À haute voix

AS : À haute voix ou à voix basse ou en silence, mais on traduit pour être lu. Alors qu'on écrit peut-être plus pour dire quelque chose, mais le traducteur, enfin l'idée de passer d'une langue à une autre "c'est qu'on lise, pas qu'on dise ", vous comprenez ? [...] Différence entre l'écriture et la traduction. Donc, je lui disais ça, «non, on ne peut pas le lire, ça, c'est pas possible, cambiamos » et on change et on change ; et devant moi j'avais cet auteur qui changeait son texte au fur et à mesure des impossibilités françaises du texte. C'était extraordinaire, non ? Quelle générosité ! Voilà.

GG : Et c'étaient des séances régulières? Vous travailliez ensemble une fois par semaine, deux fois par semaine ? Comment ça s'est passé le traintrain?

AS : Je ne saurais pas vous dire quelle était la fréquence, mais en fait nous étions face à face, et beaucoup plus proches que nous ne sommes nous, tous les deux. On était juste en face et puis il lisait " esto ", bon alors je lisais mon texte français. "No ». « Entonces - digo pues - cambia, change », et à ce moment-là on restructurait tout pour qu'en français ça soit lisible. Mais, il était évident que moi, seule, jamais je n'aurais osé faire ce que lui, auteur, faisait, il métamorphosait son texte. C'est extraordinaire. Ben, c'est une leçon, vous vous rendez compte? 
GG : Mais, mais vous aviez des discussions donc constantes sur les textes, sur la manière de les lire en français et en espagnol.

AS : Mais oui.

GG : C'était une espèce d'atelier de traduction?

AS : Mais, exactement, exactement. Ça ne pouvait pas être autrement parce que c'était le texte, mais c'était incroyablement enrichissant pour moi, surtout le fait de se rendre compte qu'un auteur, c'est hyper généreux parce qu'il va faire ce qu'il faut, il n'est pas du tout accroché à son histoire. Il est capable de transformer ce qu'il a écrit pour que ce soit accessible à d'autres, c'est-à-dire à d'autres langues.

GG : C'est surtout quand on lit votre traduction de Sarduy, pas seulement un travail de restructurer le roman, de changer les paragraphes ou de transformer la structure générale de l'œuvre, mais c'est aussi un travail sur la syntaxe. J'étais très impressionné par la manière dont vous avez réussi à tourner des phrases qui étaient impossibles en français pour les rendre lisibles en français.

AS : Voilà, voilà, voilà. La syntaxe c'est vraiment le fondement de la traduction parce c'est la construction, c'est comme quand on fait une maison, il faut bien faire des fondations et puis il faut mettre des pierres. C'est ça la syntaxe. La langue, comment elle se dit? C'est pas que des mots. Ce sont des mots qui se mettent en place. Or, c'est le "en place" qui est l'essentiel dans la traduction. C'est certainement ça.

GG : Les difficultés avec Reinaldo Arenas devaient être d'une nature très, très différente. J'imagine qu'il s'agissait surtout de questions de vocabulaire, j'imagine des termes cubains par exemple dans La Plantation, l'utilisation d'un certain nombre de noms qui sont propres à la langue cubaine. Est-ce que vous avez utilisé des dictionnaires de cubanismes? Comment vous faisiez avec ces termes?

AS : Alors, j'avais eu la chance de traduire Don Julian et le début de Don Julian se passe dans la plantation du grand-père, enfin de l'ancêtre de Juan. Et Juan m'avait donné un diccionario de cubanismos. Alors, grâce à ça, j’ai à peu près réussi à savoir, à connaître tous les mots qu'utilisait..., comment il s'appelle?

GG : Arenas

AS : Arenas, Reinaldo. Et nous nous parlons de Reinaldo parce que je suis très proche de Margarita Camacho et de son mari qui ont été les parents, si je peux dire...

GG : Et les légataires...

AS : Non seulement les légataires, mais du vivant de Reinaldo, ils ont été ceux qui l'ont aidé à sortir, si vous voulez, son œuvre de Cuba, puis des ÉtatsUnis. Donc, c'est pour ça que je dis Reinaldo. Reinaldo, c'était différent, c'était beaucoup plus poétique. Alors que chez Severo ce n'est pas poétique 
du tout. C'est rocailleux, c'est baroque, c'est baroque. [...] Chez Reinaldo $L a$ Plantation, le premier [texte] que j'ai traduit, El central [...] ça s'appelait central, [...] ça veut dire la plantation, c'était un cri poétique. Et je dois vous dire que j'ai des souvenirs épouvantables de la première traduction, parce qu'ensuite je l'ai reprise et ça a été republié aux Mille et une nuits en 2006. Mais ma fille était en train de mourir, je vous dis tout, ma fille était en train de mourir et c'était donc en en octobre 1983, elle est morte novembre 1983, 1982, pardon, et je devais rendre à François Wahl, vous savez qui était François Wahl ?

GG : Absolument.

AS : C'était un des directeurs du Seuil. Et il me disait « ça, c'est pas bien, ça c'est pas bien, là il faut reprendre " et moi qui fondais en larmes pour mille et une raisons et il me regardait «ne pleurez pas, ne pleurez pas ». Ah, c'était épouvantable. Donc, cette première traduction de La Plantation n'était pas aussi bonne qu'elle aurait pu l'être. Heureusement, j'ai eu la chance de pouvoir la corriger [...], de la reprendre pour les Mille et une nuits en 2006.

GG : Et vous avez traduit aussi les nouvelles de Fin de défilé pour les Presses de la Renaissance?

AS : Oui, ça c'était bien après. Et c'était plus facile parce que c'était linéaire, c'était narratif, si je puis dire, ça n'était pas exclusivement poétique. Et là, j'ai eu du bonheur, je me souviens avoir traduit avec bonheur ce texte. Il était, comment dirais-je, accessible. C'est formidable du texte accessible.

GG : Absolument. Pourquoi ce changement du Seuil aux Presses de la Renaissance? Vous saviez?

AS : À Fayard.

GG : À Fayard, oui.

AS : À Fayard. Pourquoi ? C'est Juan. Ça doit être un truc d'histoire avec Severo, la mort de Severo. Vous savez, les gens. Juan est passé de Gallimard au Seuil, de Seuil à Fayard et j'étais avec lui.

GG : Reinaldo Arenas aussi.

AS : Et Reinaldo pareil.

GG: Vous avez eu l'occasion de rencontrer Reinaldo Arenas à Paris?

AS : Une fois. Il était chez Pivot. Et il était déjà très très très malade, mais il a quand même réussi à se maintenir pour la soirée chez Pivot et j'étais parmi le public. Donc, je lui dis « Hola, buen... », et il m'a dit « Me gusta mucho tu ritmo, me vas a traducir, j'aime beaucoup ton rythme, tu vas traduire mon prochain livre». Alors, j'ai dit « oui, oui », mais bon.

GG : Vous saviez déjà qu'il était malade?

AS : Je crois que oui. Je ne suis pas absolument sûre. Je ne savais pas qu'il était à ce point malade et il est mort quelques mois plus tard.

GG : Absolument. Vous avez traduit les deux derniers romans de Sarduy Pour que personne ne sache que jai peur, Cocuyo, et le roman posthume de Sarduy aussi, Les oiseaux de la plage paru en 1994. La critique va parler de ces 
deux livres comme des exemplaires ou des exemples du baroque funéraire de Sarduy et qui sont loin du climat un peu plus festif du premier roman de Sarduy, en particulier de Colibrí. Et Sarduy est mort en juin 1993 et votre traduction paraît une année plus tard, en I994. Est-ce que vous avez eu le temps de discuter avec lui sur cette traduction ? Est-ce que vous avez pu travailler ensemble comme vous aviez l'habitude de le faire ? Et finalement, quels sont les souvenirs que vous gardez de votre collaboration avec lui?

AS : Alors, plusieurs questions. J'ai pu travailler avec lui pour l'avant-dernier, c'est-à-dire, Pour que personne ne sache que j'ai peur et là c'était un texte qui était beaucoup plus apaisé déjà, il avait mûri tout simplement. Il n'était plus dans cette folie baroque où il était et là, je veux dire, son texte et le mien furent à peu près pareils, pour employer le passé simple, voilà.

GG : Vous avez grandi ensemble pour ainsi dire?

AS : Peut-être, peut-être que c'est ça oui. C'est sans doute ça. Et ensuite pour le dernier, je pense que François Wahl a eu une grande importance parce que le dernier en fait n'était pas constitué quand Severo est mort. J'ai juste un tout petit souvenir terrible et merveilleux.

GG : C'est un roman en réalité inachevé?

AS : Voilà. Mais, juste avant qu'il ne meure, si je peux dire juste un petit mot, on s'est retrouvés justement pour parler de traduction.

GG : Vous l'avez vu?

AS : Ah oui, bien sûr. Et moi je ne savais pas qu'il était malade et il ne le disait pas. Il, ça ne se voyait pas encore et alors on était à table comme ça, dans un bistrot quelconque.

GG : Chez Gallimard?

AS : Non, non, non, non dans un bistrot comme ça on parlait et alors, il buvait et je lui dis : "Tengo sed, dame», il me dit : « ¿Y si tengo sida, qué ? », je me suis dit " ¿y qué importa? », tac et je bois. Je ne pouvais pas imaginer qu'il l'avait. Et puis de toute façon, ça ne se passe pas comme ça, je veux dire. Il m'a laissé boire et je l'ai aimé, parce que je me suis dit «il l'avait », mais enfin voilà, c'est un tout petit souvenir extraordinaire que j'ai de Severo.

GG : Vous avez été surprise par sa mort?

AS : Ah oui, et très attristée parce que c'était quelqu'un qui emportait le morceau, je veux dire, il vous embobinait tout de suite, il était adorable, il vous embrassait. Je me rappelle que le soir où j'ai obtenu le prix, je ne sais plus quel prix littéraire, le seul d'ailleurs que j'aie jamais eu, et donc Severo faisait partie du jury. Mais au lieu que nous dînions tous avec les membres du jury, il m’a dit : «¡Vente, vente! » et on est partis dîner tout seuls et je ne me suis pas rendu compte que c'était une injure vis-à-vis de l'ensemble du jury, mais non «iVente, vente on va tout seuls, on va tout seuls!». Moi, j'étais toute contente d'être avec lui. 


\section{Juan Goytisolo : rencontre, traduction et collaboration (Bénédicte Vauthier)}

BV : Chère Aline Schulman, passons maintenant à votre travail de médiation de l'œuvre de Juan Goytisolo pour le lecteur francophone. Gustavo vient de nous rappeler que c'est Juan Goytisolo qui vous a demandé de traduire Reivindicación del conde don Julián. Cette traduction date de 197I et c'est la première d'une collaboration qui s'étend sur quarante-cinq ans, car, sauf erreur de ma part, Gustavo Guerrero a parlé de onze titres, de douze titres, j'avais pour ma part relevé onze titres traduits (votre traduction de Beauté n'a pas de loi est de 20I6) et vous avez travaillé dans trois maisons d'édition différentes (Gallimard, Seuil et surtout Fayard). Alors, tout d'abord et avant de rentrer dans ce travail de traductrice, est-ce que vous pourriez nous parler un peu plus longuement de votre première rencontre avec Juan Goytisolo, de la découverte de son œuvre, de ses premiers romans? Comment s'est construite votre relation ? Et aussi et surtout votre travail collaboratif, vous venez de nous parler de votre travail en collaboration avec Severo Sarduy. Comment s'est construit ce travail collaboratif avec Juan Goytisolo, dont vous avez fait état dans le prologue à Don Quichotte? Pouvez-vous nous l'expliquer de manière un peu concrète? Et puis comment a évolué ce travail collaboratif?

AS : Donc, comme je l'ai dit tout à l'heure, le point de départ, c'est cette rencontre incroyable de nos deux mémoires, c'est-à-dire lui étant à Tanger et écrivant à partir de Tanger, moi ayant vécu à Tanger et quand j'ouvre la première page de Señas de identidad, pardon de Don Julian, imaginez-vous pour une traductrice novel parce que c'était avant le Donoso, c'était juste après quelques petits poèmes de Cernuda, alors vous voyez comment on est un petit peu pas sûre de soi, vous pouvez imaginer, et j'ouvre cette page «Tierra ingrata, espuria y mezquina, jamás volveré a ti » et puis je continue et je me dis «non, eh eh, je rêve ", je continue encore et je rêve encore plus. C'était exactement ce que moi, la vision que moi j'avais pendant les trois années où nous avions vécu à Tanger au retour d'Argentine. Je voyais l'Espagne, c'està-dire, je voyais un truc en face, je ne savais pas ce que c'était, mais c'était un truc qui allait, qui venait, alors que se borraba, qui s'effaçait, et puis qui revenait selon le temps, selon les nuages. Et puis après quand il parle du grand soco, du zoco chico, zoco grande, enfin tout ça, mais moi, tous les vendredis, on nous amenait de l'école, nous allions nous promener en rangs serrés dans les zocos, etcetera. Alors, les cinémas dont il parle, moi j'y étais, vous comprenez ? Ce n'est pas possible. Alors du coup, comment vous dire, il y a eu une rencontre, moi j'avais oublié le Maroc quand j'ai retrouvé Juan, à l'époque où il me l'a demandé, et tout d'un coup c'est comme si c'était un passé apocryphe qui revenait comme ça à travers les souvenirs d'un autre. C'est moi-même qui me reconstituait. Alors soixante-seize pages, les premières 
soixante-seize pages sans un point, que des deux points et pas un verbe à la forme pronominale, que des gérondifs. Bon, alors, essayez d'imaginer une traductrice totalement novel, alors je vous explique un petit peu. Donc, je me suis dit «durmiendo, cantando, corriendo ", c'est magnifiquement bien, comment dirais-je, c'est sonore, c'est accentué sur l'avant-dernière syllabe. Je ne pouvais pas moi en français dire « mangeant, dormant, pissant ", etcetera. C'était impossible, impossible. Alors, qu'est-ce que j'ai fait ? Bon, je me suis dit, il faut changer quelque chose. Les deux points on les laisse, mais je vais mettre des infinitifs. Alors, je ne sais pas s'il y a peut-être aujourd'hui des gens qui se souviennent de qui était Monsieur Molho, el señor Mauricio Molho, que nous avons eu nous comme professeur et qui nous expliquait (mais je vais vous le danser), qui nous expliquait le gérondif. Alors, Molho dansant le gérondif. Molho boitait et alors... Le gérondif c'est un temps qui se mord la queue. Oups. (Aline Schulman se lève et esquisse quelques pas de danse.) Et il continuait, attendez, comme ça. Aucun des étudiants de Monsieur Molho n'a jamais oublié ce qu'était le gérondif et comment le gérondif se mordait la queue. Bon je me suis dit, on va mettre plutôt qu'un gérondif qui évidemment est porteur de temps, un infinitif, l'infinitif ne porte pas de temps, étant achronique, mais dans la mesure où il n'y en a pas, le lecteur peut en mettre autant qu'il veut. Et je me suis dit « comme ça, ça marche » et je n'ai mis que des infinitifs pendant soixante-seize pages au lieu de ne mettre que des gérondifs. Et alors, j'ai eu le bonheur de m'apercevoir que dans le livre suivant Juan sin tierra, Juan au lieu de mettre des gérondifs, eh ben il mettait des infinitifs, en espagnol, ah. Donc, pour moi c'était beaucoup plus facile, mais il s'était peut-être rendu compte que c'était plus léger aussi. Voilà, ça c'est ma première, comment dirais-je, ma première tentative, si vous voulez, d'appropriation du texte de Juan. Alors, pour tout ce qui était labyrinthique etcetera, je l'ai suivi, comment vous dire, pour traduire, il faut être en confiance, si on ne comprend pas, parce que je n'ai rien compris. Je vous dis tout de suite, je n'ai rien compris à ce que, lui, voulait dire dans l'ensemble du texte ; et aujourd'hui encore, bon, c'est juste. Mais, mais pendant que je traduisais, vous savez je ne lis jamais à l'avance, parce que si je lis c'est fichu. Il faut découvrir pendant qu'on traduit. Je n'ai jamais su vraiment où il voulait en venir, mais j'avais confiance et je le suivais, si vous voulez, dans son parcours labyrinthique parce que je le suivais dans les souks. Si vous voulez, ce parcours labyrinthique, pour moi il était naturel. Et cette confiance que j'avais en lui, en son écriture, je savais qu'on partageait un même paysage imaginatif et donc j'y allais, voilà.

BV : On va revenir encore sur le travail de traduction de Don Julian et je me permets de revenir un peu en arrière, sur ma première question, qui était une question peut-être aussi presque personnelle, mais c'est vrai que dans votre réponse, la personne de Juan c'est l'œuvre de Juan, c'est-à-dire 
que vous avez commencé directement. Est-ce que vous aviez lu l'œuvre de Juan Juan Goytisolo avant même d'en faire la connaissance ou vous l'aviez connu, rencontré et puis vous l'avez lu ? Est-ce que vous le connaissiez et puis vous l'avez rencontré ? Est-ce que vous avez mis un livre sur un visage ou un visage sur un livre? Quel était le sens de la relation ? Vous avez évoqué tout à l'heure votre travail sur Cernuda en compagnie de Robert Marrast. Comment se fait ce premier contact, est-ce avec l'œuvre ou avec Juan Goytisolo ? Est-ce que vous en avez encore le souvenir?

AS : Alors, bien sûr. Vous savez, on n'oublie pas ces choses-là. Moi, j'avais lu Juegos de mano comme tout le monde. Eh, c'était un livre qui était paru, je crois, en 1958 ou [...]

BV : 1954 en espagnol, 1957 en traduction.

AS : Mais, je crois que je l'avais lu en espagnol, enfin c'était un livre qui avait fait du bruit et donc je connaissais Goytisolo comme ça, mais pas plus, et cette page [...] quand je me suis aperçue qu'au fond le Maroc pour lui, c'était sa nouvelle terre d'adoption, comment vous dire, Juan a eu un parcours différent. Moi, je suis née au Maroc et je suis partie en France, lui il est né en Espagne et il est venu au Maroc, mais il y avait tout de même, même si, comment dirais-je, le parcours était inverse, il y avait un lieu de retrouvaille essentiel. Et je crois que c'est ça qui était important. Non, je ne le connaissais pas à part Juegos de mano.

$\mathrm{BV}$ : Et dans ce premier travail, justement pour faire un peu un retour sur le passage de Juan Goytisolo chez Gallimard et nous attarder maintenant sur Don Julian. Au début des années I970, donc, vous prenez le relais de MauriceEdgar Coindreau, qui a traduit l'œuvre de jeunesse de Juan Goytisolo, mais qui a aussi traduit le premier volume de ce qui sera par la suite vu comme une trilogie, non ? Les fameuses Pièces d’identité. Maurice-Edgar Coindreau qui est non seulement le traducteur dans un premier temps de Juan Goytisolo, mais aussi de la génération des enfants de la guerre ou la génération du demisiècle, qui est aussi le promoteur de cette collection «Du monde entier » en langue espagnole, comme le démontre sa correspondance chez Gallimard. Comment a-t-il vécu le fait que vous deveniez la traductrice de ce second volume ? Est-ce que c'est Juan Goytisolo qui vous l'a demandé ? Est-ce que c'est vous justement dans ce rapport d'appropriation, de reconnaissance de son œuvre qui lui avez demandé si vous pouviez le traduire? Est-ce qu'il y a eu un conflit? En avez-vous le souvenir?

AS : Si, si, je me souviens très bien. Je me souviens très bien, qu'il n'y a pas eu tout ça. Voyez-vous, Juan quand il a vu comment je traduisais, en particulier « Diré como nacisteis ", Los placeres prohibidos, enfin, les poèmes de Cernuda. Je n'ose pas vous dire la vérité mais je parle de cet ami compositeur que j'appelle Chano et la plupart des poèmes, c'est lui qui me les avait traduits ; seulement, ça personne ne le sait, il n'y a que vous et moi. 
Chano est mort, donc voilà. Comment vous dire, je prenais tout ce que je pouvais partout. Il faut dire, quand on est neuf comme ça, on s'alimente de ce que l'on peut. Maintenant, Coindreau je ne l'ai jamais vu. J'étais toute étonnée que Juan... Après j'ai compris, j'ai compris. Coindreau n'était pas seulement traducteur d'espagnol. Il était surtout traducteur d'américain. C'est lui qui a fait venir Faulkner et si Faulkner a eu du succès aux ÉtatsUnis, c'est parce qu'en France il a eu un succès extraordinaire. Et donc il y avait Faulkner, il y avait tous les grands quoi. Et l'anglais est une langue beaucoup plus raide que le français, que l'espagnol. Or, Juan ce qu'il aimait dans l'espagnol c'était les assonances, c'était, si vous voulez, ce rythme, il a toujours dit : «Toi, dis ce que tu veux, du moment qu'il y a du rythme ». Et il m'a dit «Coindreau no tiene, n'a pas le rythme qui moi me conviendrait, or je sens que toi tu l'as ». Et c'est là-dessus qu'on s'est reconnus, bien avant de se reconnaître dans le territoire, si vous voulez. Voilà comment ça s'est passé.

BV : D'accord. Je crois que peut-être je m'arrête un moment sur cette question du rythme [...] Parce qu'en écoutant les réponses que vous évoquiez avec Gustavo Guerrero sur Severo Sarduy, vous avez parlé de musique, de réalité, puis vous avez parlé de visualisation. Vous vous êtes vue à Tanger; et puis vous avez fait l'opposition entre traduction du narratif et du poétique, ce qui rejoint la dimension baroque et donc [...] je me demande dans quelle mesure cette appréhension presque synesthésique de la traduction non pas par le graphique, mais par l'oreille (et je pense que c'est une dimension très importante pour Juan Goytisolo qui se lisait, je veux dire cette lecture à voix haute). Est-ce que vous pouvez nous en dire un peu plus ? Ça m'a vraiment frappé en vous écoutant maintenant, la vue, l'oreille, et puis en même temps vous avez parlé d'être lue. Donc comment conjuguer ces trois dimensions du lisible, du visible, de l'audible pour une traductrice?

AS : C'est la musique. La musique peut être visuelle aussi, comment vous dire, il y a cette visualisation c'est une chance que j'ai eue dans ma vie de visualiser les premiers, mais après quand j'ai traduit Reinaldo il n'y avait pas de visualisation, mais il y avait une musique, donc, comment vous dire, on a plusieurs cordes à notre arc, en tant que traducteurs, comme vous dites, l'oreille, je dirais l'odorat on laisse de côté, mais on a l'oreille, on a la vue et on a la parole, et selon les moments, quand on peut joindre les trois, c'est le bonheur absolu et la traduction s'en ressent dans le bon sens du terme. Quand on ne peut pas, on fait le choix de l'un ou de l'autre. Alors, chez Juan la musique ce n'était pas de la grande musique, voyez-vous, tandis que chez Reinaldo, oui, c'était de la grande musique, et dans les autres aussi, mais Juan avait envie que ça tombe bien, voyez, une espèce de trois quatre, comme ça, pom pom pom pom pom pom, les assonances le gustan mucho, il aimait, il aimait beaucoup les assonances, alors il fallait faire les assonances en français voyez, pas des rimes mais des assonances et ce n'est pas facile en 
français et lui, il aimait ça, je ne sais pas comment vous dire [...] puis il a écrit des poèmes aussi, mais on ne peut pas dire que sa prose ait été poétique ; mais il avait lui l'impression qu'il fallait rendre quelque chose au-dessous ou au-dessus des mots et de cette libido permanente. Voilà. Est-ce que je réponds [à votre question] ?

BV : Oui, oui, mais je pense que ça va nous permettre d'enchaîner et puis de rester encore un petit moment sur Don Julian parce que quand on publie la traduction en français, elle est accompagnée d'une préface, d'un prologue de Josep María Castellet dans lequel le critique catalan met en évidence le fait que ce n'est pas seulement un roman palimpseste, c'est un roman fragmenté, c'est un roman pour moi d'une certaine manière poétique en ce sens, ces larges isotopies, même si vous dites que vous le découvrez en cheminant dans les labyrinthes et les souks, mais il parle aussi de ce roman comme " critique de l'historiographie espagnole", comme « introduction à l'histoire de l'Espagne ", de ce roman sur "l'hispanité », et ce, non pas seulement pour les hispanistes, mais aussi pour les Espagnols, qui ne connaissaient pas cette image de l'Espagne que Juan Goytisolo véhicule aussi à travers sa découverte d'Américo Castro. Comment faites-vous, et on le voit dans l'apparat critique fait par Linda Gould Levine, comment faites-vous, jeune traductrice, comme vous dites, pour relever ce défi intertextuel, défi qui met en place cette destruction créatrice à un moment où finalement nous ne savions pas et vous ne saviez pas ce que nous savons aujourd'hui dans la réception critique, dans les révélations qu'a faites Juan Goytisolo sur son propre travail de création? Comment faire face à ce que cinquante ans plus tard [reconnaît la critique] ? Je reconnais que je suis sans voix quand j'imagine le peu de recul que vous aviez pour traduire un livre d'une telle complexité et, qui plus est, quand vous nous dites « c'est mon deuxième texte après ces quelques traductions de poèmes ». Comment relever ce défi ?

AS : C'était ou infaisable ou possible. Je m'explique parce que ça n'a pas l'air très malin. Vous savez, Bonnefoy dit, et je l'ai retenu parce que c'est vraiment magnifique, « la traduction ce n'est pas pensée, mais action », c'est tout. J'ai traduit Don Julian sans comprendre, comme je vous l'ai dit, le sens total où il voulait en venir, ben je voyais bien quand il se moquait, je voyais bien quand il ne se moquait pas, je veux dire, l'ironie, le sarcasme surtout, plus que l'ironie comme on a dit tout à l'heure, mais " atenerse a las palabras ", je tenais le fil, si vous voulez, mon fil d'Ariane, c'était la phrase et ce fil d'Ariane, en plus, il y en avait un double, si vous voulez, il y avait le fil d'Ariane des souks, il y avait le fil d'Ariane de tout ce background, comme on dit en anglais, dont il parlait et que moi je connaissais tout à fait, donc je n'étais pas perdue, si vous voulez. J'étais perdue dans le sens global du texte, mais je m'en fichais pas mal. Ça ne m'intéressait pas, si vous voulez, mais je n'étais pas perdue dans le déroulement du paragraphe, dans le déroule- 
ment de tout ce qui était en train de se faire dans le texte. C'est ça, qu'il faut comprendre. On est dans le mot. Il faut reconnaitre, comment dirais-je, un déroulement. Je ne sais pas comment le dire autrement. C'est la syntaxe, et quand cette syntaxe vous rappelle des lieux, vous rappelle encore une fois le labyrinthique, le sournois, le le..., je ne sais pas comment vous dire.

BV : D'accord. Alors, peut-être une dernière question un peu précise sur ce même roman. Vous avez choisi, donc vous ou vous et/ou l'éditeur le titre Don Julian pour la traduction de ce roman dont le titre original était Reivindicación del conde don Julián. Est-ce que vous vous souvenez si c'est vous qui avez proposé ce changement de titre qui est un changement conséquent, changement que Juan Goytisolo finira par adopter, mais quinze ans plus tard ? Et il dira à ce moment-là : " Je n'ai plus besoin de la revendication ». Alors, est-ce que vous vous souvenez du pourquoi de cette concision ? Pourquoi faire disparaître ce qui à l'époque était une revendication ? Est-ce que vous en avez le souvenir ? Est-ce que Dionys Mascolo a pu participer à cette proposition du changement de titre incisif en français? Les lecteurs français ne vont pas comprendre pourquoi la revendication. En tout cas, je suis interpelée [au sujet] du caractère soit prémonitoire, soit prophétique du choix de la traduction en français que rejoint par la suite l'original espagnol.

AS : Alors, je vous le dis tout simplement et honnêtement, je n'y suis pour rien. J'étais une traductrice novel et il n'était pas question qu'on me demande quelque chose, qu'on me demande même mon avis sur le titre qu'il fallait mettre. Ah non, comment vous dire, je suppose que ça s'est passé entre Juan et son éditeur Gallimard, voyez. Moi, je n'ai rien eu à dire, je vous le dis aussi honnêtement et simplement que les choses se sont passées. Reivindicación del conde don Julián s'est transformé en Don Julian sans que j'en sache, que j'en connaisse la raison et moi j'avoue que ça ne me changeait pas trop. Je veux dire l'un dans l'autre et réciproquement, on n'avait pas besoin d'un titre aussi long, voilà. Moi, ça m’a semblé tout à fait acceptable et je n'ai même pas été rouspéter, comme on dit.

BV : Et quarante-cinq ans après ça vous semble encore acceptable comme traductrice et dans ce travail de collaboration ? [...]

AS : Qu'on ne me demande pas mon avis?

BV : Oui, parce que vous avez ce contact tellement proche, intime avec le texte. Donner un titre, ça a un sens pour un auteur, dans cette négociation pour vous, ça reste l'auteur?

AS : Alors, je vous donne un autre exemple, bon, dans ce cas précis Juan était là. Donc, c'était Juan qui décidait, quand même c'était son enfant, ce n'était pas le mien. Bon, j'étais une sorte de marâtre comme ça, mais là ou moi j'ai donné le titre, c'est dans le livre précédent et là Albert n'y est pour rien, Albert Bensoussan, c'était El lugar sin límites, et je me suis dit que traduire Le lieu sans limites n'était pas possible et c'est moi qui ai dit : «On tra- 
duira Ce lieu sans limites ». Là c'est moi qui suis responsable du texte, mais parce que Donoso n'était pas là, mais là quand Juan est là, qu'est-ce que vous voulez que je dise. Moi je dis amen.

$\mathrm{BV}$ : Alors, peut-être plus encore que Don Julian qui révèle déjà ce travail de destruction, d'éclatement de la "langue vernaculaire », qui se décline dans ses variétés linguistiques mexicaine, argentine et cubaine, on va y revenir, notamment sous l'impulsion du boom de la littérature hispano-américaine, Juan sin tierra, Jean sans terre puis Makbara posent des problèmes linguistiques « insolubles » ou difficilement traduisibles vers le français. En effet, ces textes sont non seulement hétérolingues, mais aussi plurilingues (français, anglais, italien, arabe, classique et dialectal) avec une dominante très marquée du français, même si c'est l'explicit en espagnol, en arabe translittéré puis calligraphié de Juan sin tierra qui a le plus souvent retenu l'attention. Et cependant, en consultant vos traductions de ces livres, j'ai pu constater que jamais, jamais - qu'il s'agisse d'un bref syntagme, de plusieurs paragraphes voire même d'un chapitre tout entier - vous n'avez opté pour un démarcage typographique - ou linguistique - du texte d'origine en français, ce qui conduit, et je me permets de citer dans ce cas-ci, comme l'observait Myriam Suchet, à une "résorption apparemment naturelle de l'hétérolinguisme » ou, pour le dire à la suite de Derrida parlant de «In Eins » de Paul Celan, à « effacer cela même que [la traduction] garde, l'effet d'étrangeté du français (sans italiques) ». J'aimerais, si c'était possible, que vous reveniez sur ce choix qui d'une certaine manière a un impact sur la perception du lecteur francophone.

AS : Alors, je vais vous dire deux choses. Une première, une plaisanterie, mais en fait une plaisanterie qui est vraie. C'est que quand je voyais des trucs en français je me disais « chic, je n'ai pas besoin de les traduire ». Alors, tant mieux s'il y en avait, plus les passages en français étaient nombreux, plus j'étais ravie, bon. Alors, ça c'est une première chose qui est à moitié une plaisanterie seulement. Maintenant, comme je disais à Gustavo Guerrero tout à l'heure et à toi aussi, je suis tombée hier soir sur une lettre de Juan me disant : «Il n'est pas question... » (parce qu'au départ moi j'avais mis des italiques), «il n'est pas question de mettre les langues étrangères du texte en italique. Toutes les langues doivent avoir le même niveau. Aucune ne doit être supérieure ou inférieure à l'autre ». Alors, voilà, tout est dit là, parce que moi, j'avais mis des italiques. Alors, évidemment, je n'allais pas mettre en français dans le texte, qu'est-ce que vous voulez, ah non ça ce n'est pas possible. Ce n'est pas possible. Et alors, attendez, aujourd'hui peut-être que Juan m'aurait dit «mets en chinois », mais à l'époque il n'y avait pas de chinois en Europe, il avait...

BV : En espagnol ? Est-ce que vous avez pensé par exemple inverser... 
AS : Non. Oui, mais alors on aurait dit : «mais, elle ne traduit pas!». Vous comprenez, non, non ; et puis c'était assez complexe comme ça, je sais que Juan jamais ne m'a dit : " mets autre chose ». Je vous dis la seule chose qu'on aurait pu mettre à la rigueur c'était du chinois ou du japonais aujourd'hui, mais à l'époque il n'y en avait pas. Alors, il y en avait bien assez. Vous ne croyez pas que c'était assez compliqué comme ça?

$\mathrm{BV}: \mathrm{Quand}$ on pense aux textes qui sont les plus représentatifs à ce point de vue, c'est Makbara ou Juan sin tierra ou même Don Julián. Et c'est vrai que la vision de ce Maroc plurilingue, polyglotte, dont faisait état Gustavo Guerrero, si vous mettiez du chinois, ça me paraitrait un roman de science-fiction. [...] Le fait que disparaisse le français, d'une certaine manière absorbé dans la traduction française, le texte perd cette dimension plurilingue du Maroc de l'époque... [...] Quand je dis «l'espagnol » [...]... je le voyais comme un reflet [...] de cette réalité dont vous faites état et donc vous êtes vous-même issue [...] et don Juan parle, quand son jeune guide l'accompagne dans le souk et quand lui-même apprend l'arabe ; il essaie qu'on ne lui parle pas en espagnol et qu'on ne lui parle pas en français. Donc, on voit cette rivalité des langues.

AS : Oui, oui, absolument. [...] Mais, voyez-vous, ça ne s'est jamais posé. [...] Il n'était pas question, jamais il n'en a été question, parce qu'évidemment on aurait pu le mettre en espagnol, mais à ce moment-là la traduction venant d'un texte espagnol pouvait... Enfin, je ne sais pas. Je n'ai pas de réponse. L'unique réponse que j'aie est ce que j'ai retrouvé dans la lettre où il n'est pas question de mettre d'italiques, dans aucune, puisque les langues sont égales, il n'y a pas de niveau. Les choses sont parfois plus simples qu'on ne pense et on ne pensait pas l'hétérolinguisme. À l'époque ça n'existait pas d'abord, ah, ce terme. C'est tout ce que je peux dire.

BV : Merci. Alors, peut-être après les romans espagnols et du Maghreb, passons à Paysages après la bataille, le roman français par excellence, parisien même, de Juan Goytisolo, et ce qui n'a pas manqué d'irriter certains lecteurs ou critiques français. Alors, comme vous avez déclaré être particulièrement attentive au lecteur, je me demandais si vous vous souveniez de nouveau, comment vous avez dû procéder pour traduire ce roman ironique, polyphonique qui est écrit dans un contexte particulièrement marqué par la montée de l'extrême droite, par le parti de Jean-Marie Le Pen et écrit aussi au départ d'un certain nombre de documents sources en français, des fragments de Libération, du Monde. Comment avez-vous fait pour rendre d'une certaine manière cette texture cette fois si contextuelle?

AS : Alors, je reviendrai une minute sur Don Julian pour vous parler justement d'une liberté que j'ai connue et qui m'a permis de traduire ensuite le Quichotte et le reste. Mais dans ce texte-là c'est là où il y a les petites annonces de Libé ? [...] Bon, alors c'étaient des annonces espagnoles. [...] il faut 
que je recommence par Don Julian. Dans Don Julian il y a un passage où don Álvaro Mendiola est en train de crever, je ne sais pas, et puis il lui faut une épitaphe. Et Juan utilise le texte de l'hymne de la légion espagnole, « en tu pecho ", no sé qué, « legionario de bravas legiones ». Ça ne pouvait absolument pas marcher en français parce que les Français ignorent le texte de ce truc-là et donc ils n'auraient pas senti l'humour et le sarcasme surtout. Alors, je me suis cassé la tête ; et puis j'ai pensé à quelque chose et ce que j'ai fait finalement c'est prendre... (bien évidemment, avec l'acceptation de Juan, parce que je lui demandais), j’ai pris un morceau de Mon Légionnaire d'Édith Piaf et une phrase de La Marseillaise et j'ai mélangé et ça a donné, justement dans le contexte dérisoire, si vous voulez, ça a donné l'équivalent, l'homologie. L'homologie, ce n'est pas la traduction exacte, mais je dois dire que c'est cet endroit-là, c'est ce lieu-là et ce moment-là de ma vie qui, après, m'a permis, m'a dit que je pouvais, oui qui m'a permis [...] de traduire par exemple « no con quien naces, sino con quien pases », « qui se frotte à l'ail, ne peut sentir la giroflée ", vous comprenez ? C'est-à-dire trouver vraiment une homologie qui frappe l'oreille de celui qui écoute et pas qui colle à l'original de départ. C'est Juan qui m'a dit : «Tu y vas ».

$\mathrm{BV}$ : Alors, c'était une question que je voulais réserver pour la fin mais je pense qu'elle [...] est directement liée à ce que vous venez d'évoquer. Je me suis demandé, ou je me demande en vous écoutant, si cette volonté, cette dimension homologique n'est pas aussi liée à un regard que la langue française a sur les langues étrangères. Je l'ai perçu en lisant des traductions du russe, je pense à Kristeva traduisant Bakhtine, je pense à la traduction de la littérature francophone. Est-ce qu'il se passerait la même chose dans d'autres langues ou est-ce une attitude du français, [une attitude] centralisatrice ? Est-ce que vous êtes sentie parfois [...], comme traductrice, dans un rapport de la langue française aux langues étrangères, dans un rapport qui fait pression?

\section{AS : Domination?}

BV : Oui, qui vous impose d'une certaine manière cette volonté ? Pourquoi ne pas laisser ce caractère d'étrangeté ? Par exemple, vous venez de dire, il faudrait mettre Piaf parce que le lecteur francophone ne va pas comprendre. Est-ce que le lecteur espagnol comprend d'emblée ? Est-ce qu'il n'est pas lui-même conduit à faire un cheminement?

AS : Je pense qu'il comprend parfaitement qu'il s'agit d'un hymne que Juan abhorre et que ce qu'il essaie de faire c'est justement une épitaphe ridicule, si vous voulez. Alors, je me suis dit que si je traduisais par les mêmes mots, les lecteurs français ne comprendraient pas. Ce qui compte, c'est de comprendre. Quant à l'hégémonie, si je puis dire, de la langue française, n'oubliez pas que je suis une trilingue de naissance, et que, je dois l'avouer, je n'ai pas une goutte de sang français dans les veines. Je suis obligée de le 
dire, " y a mucha honra », comme on dit. Mais bon c'est comme ça, donc pour moi l'hégémonie de la France oui et non, enfin, je veux dire, vous ne tombez peut-être pas tout à fait sur la personne qu'il faut [...]. Ce qui compte surtout c'est la réception. Pour moi la réception est essentielle. Il faut savoir, comme je l'ai écrit quelque part, mesurer le respect que l'on doit à l'auteur et au lecteur. Le lecteur, c'est quand même lui qui va recevoir. Or, il faut qu'ils reçoivent les mêmes chocs. Les mêmes amusements, les mêmes chocs, les mêmes tristesses, les mêmes joies, la même musique, si possible évidemment, mais on fait ce qu'on peut, voilà.

BV : Merci. Et pour revenir sur Paysages [après la bataille] - je vous ai interrompue -, et par rapport à cette idée-là, c'est un contexte français, mais il n'y avait pas nécessairement beaucoup de langues françaises dans Paysages après la bataille. Traduire un livre qui a cette fois comme contexte, comme arrière-fond, un univers français, parisien, un univers que Juan Goytisolo remet en question, est-ce que pour vous cela a représenté un autre défi, si on pense à l'intertextualité, comme j'ai dit, très hispanique de Don Julián ? Pour moi Paysages est un roman très français, très parisien. Est-ce que ça représente un autre défi pour la traductrice?

AS : Je ne crois pas, d'autant que, si vous voulez, je visualisais aussi parfaitement tout ça puisque j'allais chez lui, vous comprenez, et il habitait le quartier qu'il décrit dans Paysages. Pour moi c'était aussi familier que les souks. Alors, vous savez c'est la chance d'avoir eu ce rapport, cette visualisation possible, donc, que ce soit les souks ou la rue du Sentier, pour moi c'est à peu près la même chose parce que Juan est là au milieu.

$\mathrm{BV}$ : Je vous poserai une dernière question, une question qui servira aussi de transition vers l'intervention de Roland Béhar qui va s'entretenir avec vous de votre travail de retraduction des classiques espagnols - un travail qui a n'a pas seulement été applaudi par Juan Goytisolo, il faut le souligner, mais qui l'a même amené à préfacer votre travail de traductrice. Je me suis demandé si vous n'aviez jamais souhaité écrire une note de traductrice pour accompagner les textes contemporains que vous avez traduits en en signalant les enjeux? Plus concrètement, pourquoi avoir rendu votre présence de traductrice si discrète, voire même invisible, après avoir déclaré, et je vous cite cette fois, " avoir découvert en Severo Sarduy et Goytisolo des auteurs non pas avares, mais généreux ", vous l'évoquiez tout à l'heure, " capables de sacrifier la forme première pour préserver, dans la traduction, l'effet premier, le rapport premier ». Je continue à vous citer : "Ils vous ont toujours encouragée à respecter leur texte au maximum, c'est-à-dire à le transformer, à le refigurer afin de donner au lecteur français l'équivalent le plus proche, parfois très éloigné du texte original [...]. Au pire, une petite phrase : "Les modifications apportées au texte original ont été faites en accord avec l'auteur"». Alors pourquoi d'une certaine manière écrivez-vous « au pire »? 
AS : Parce que j'ai horreur de ça. Simplement j'aurais pu dire " au mieux », mais ce n'est pas mon opinion. [...] «Au pire», c'est quand on ne peut plus faire autrement. Autant le dire, parce que sinon les gens vont penser que je fais ce que je veux et que l'auteur n'a pas son mot à dire. Or, l'auteur est tout à fait en accord avec moi, c'est pour ça que je dis « au pire », voilà.

BV : Et ça rejoint peut-être l'une des questions que vous a posée tout à l'heure Gustavo. Est-ce que vous vous sentez parfois co-auteur, co-autrice justement? Cette distinction. Tout à l'heure vous parliez de la traduction comme brouillon. [...] Sur les livres de Severo Sarduy figurait « en collaboration avec l'auteur ", dans le cas de Juan Goytisolo cette mention n'apparait jamais, mais on voit aussi que ce travail a été très présent, très prégnant. Vous avez aussi parlé des effets rétroactifs, la syntaxe de Juan qui adapte, qui passe du gérondif à l'infinitif. Est-ce que vous avez eu ce sentiment ? Est-ce que pour vous ce sont eux qui sont responsables? Vous avez l'air, quand vous le présentez, de dire : "ce sont eux ", et puis dans votre travail il y a aussi un véritable travail de création, pour le lecteur francophone, qui sent cela...

AS : Comment vous dire ? Vous savez, le travail de traduction, jusqu'à un certain moment, je me souviens qu'à la fac, on me dit «mais qu'est-ce que tu fous là ? Pourquoi tu perds ton temps à traduire ce pauvre type ?», vous comprenez, à l'époque... Aujourd'hui tout le monde traduit, mais à l'époque, c'était vraiment une perte de temps sèche, c'était pas du tout un travail reconnu et je vous avoue que moi, tant que j'ai traduit Juan, je ne me sentais aucunement auteur, ni co-auteur, j'étais là et puis je l'aimais beaucoup et puis ça me permettait, si vous voulez, ça a l'air idiot de dire ça, mais c'est vrai, à travers la traduction j'avais accès à des êtres merveilleux [...] Donc je n'avais pas besoin d'aller les chercher, ils venaient vers moi. Donc à travers Juan, j'ai connu toutes sortes de gens formidables, dont Monique Lange, qui était sa femme, et je me contentais. Jean Genet, même Jean Genet, qui me regardait d'un air terrible, je me contentais de ça, c'était ça mon cadeau. À travers eux, à travers ces livres que je traduisais, d'auteurs qui étaient des gens merveilleux, je pouvais connaitre des gens fantastiques que moi-même toute seule je n'aurais jamais connus. C'était ça surtout. La traductrice ; ce n'était rien à l'époque; et il a fallu attendre le succès, si je puis dire, de Don Quichotte pour que je me sente enfin reconnue pour ce que j'avais fait, si vous voulez, sinon bof. Ce n'est rien, je l'assure.

\section{Les classiques : Cervantès, Rojas, Quevedo, Thérèse d'Avila (Roland Béhar)}

RB : Comme cela a déjà été évoqué, vers le milieu des années 1990 vous avez été amenée à traduire des classiques de la littérature du Siècle d'Or: d'abord, Don Quichotte (Le Seuil en 1997), puis La Célestine, de Fernando de 
Rojas (Fayard, 2006), puis le Buscón de Quevedo, et enfin une Anthologie de textes de Thérèse d'Avila, Les chemins de la perfection (Fayard, 2015). Ces traductions forment un ensemble qui pose une série de questions générales, que je voudrais d'abord formuler rapidement, avant d'envisager plus en détail votre traduction de chacune de ces œuvres, et en particulier du Quichotte. Alors, tout d'abord, quand on considère la trajectoire très riche, qui vient d'être évoquée, de vos traductions depuis la fin des années 1960, on est tenté de s'interroger sur les raisons qui vous ont fait aborder les rives de l'espagnol classique, et qui plus est en commençant par le texte peut-être le plus monumental de tous, Don Quichotte de la Manche. Je voudrais vous poser d'abord deux questions à ce sujet : premièrement, comment la proposition vous a-t-elle été faite de traduire Don Quichotte ? Quel fut en particulier le rôle de Sarduy, pour l'idée même d'une traduction à nouveaux frais du Quichotte, et celui d'Annie Morvan, alors directrice du domaine hispanique aux Éditions du Seuil ? Et en second lieu, est-ce que cette traduction était le résultat d'un concours de circonstances, ou répondait-elle à une envie ancienne, nourrie peut-être au fil des années, parallèlement aux traductions d'autres textes que vous alliez publiant - je crois me souvenir en particulier qu'il y avait eu, en amont, un épisode lié aux Tréteaux de Maître Pierre de Manuel de Falla?

AS : Alors, vous m'avez posé plusieurs questions. Je commencerai par la première, comment cela s'est-il fait ? Vous savez c'est comme l'histoire de « puis si tu fais médecine, on se verra plus, fais espagnol », là, c'est tout d'un coup, je crois que c'était l'heure du déjeuner ou juste après déjeuner, je crois qu'on était encore à table, j'ai un coup de téléphone d'Annie : « dis donc tu veux traduire le Quichotte? ». Évidemment, j'ai cru que c'était une plaisanterie et elle m'a dit " mais non, mais non, c'est vrai, c'est vrai ». Je dis « qu'est-ce que c'est que cette chose-là ». Enfin, bref, voilà, c'était juste une question d'Annie Morvan qui était alors comme tu l'as dit éditrice de l'espagnol au Seuil et cela venait sans doute quelque part de Severo, mais moi je n'ai jamais eu accès aux racines, si vous voulez, de ce désir du Seuil. Simplement, Annie Morvan m'a expliqué : «le Seuil voudrait une traduction du Quichotte sans notes et qui ne tombe pas des mains » et alors on m'a dit une chose, elle ne savait pas à quel point elle pouvait tomber juste, « et que Nathalie Sarraute puisse lire ». Parce qu'on savait tous que Nathalie, dès qu'elle lisait le Quichotte, ça lui tombait des mains. Or moi je connaissais très bien, par des hasards de la vie, Nathalie Sarraute et alors je lui ait dit : «Voilà Nathalie, malheureusement j'ai fini ». Mais elle avait 99 ans, et elle ne pouvait plus lire.

Mais enfin, voilà ce qu'on m'a dit au Seuil : " Une traduction moderne, sans notes ", enfin, "sans notes " c'est plutôt moi qui l'ajoute, mais " une traduction moderne que Nathalie Sarraute puisse lire». Alors, évidemment 
j'ai cru véritablement à une plaisanterie. J'ai demandé si ça n'avait pas été demandé à d'autres avant moi, on m'a dit que oui, mais qu'ils avaient refusé. Et j'étais en train de faire une thèse que je n'aurais jamais finie et je me suis dit « autant faire le Quichotte » et puis je me suis dit aussi «surtout je peux toujours commencer, je n'ai pas besoin de finir ». Voyez, voilà enfin, toute une série de, comment dirais-je, de parois, de parois de verre, qu'on se met devant les yeux en se disant "c'est fini, on arrête, c'est pas la peine ", voyez. Et qu'est-ce que je risque surtout à essayer ? C'était ça surtout, qu'est-ce que je risque ? Rien. Je peux toujours m'arrêter quand je voudrai. Je rends l'argent et on s'en va, voilà. Et sachant que je ne risquais rien et que c'était quand même mon métier, j’ai dit oui. Voilà, tout simple.

RB : Est-ce qu'il y avait une familiarité avec le texte antérieure à cette traduction?

AS : Alors, je peux vous dire que je l'ai eu à l'écrit et à l'oral. À l'écrit, il y avait « Maîtres et serviteurs » dans le Quichotte et La Célestine, et à l'oral, j'ai eu « L'art du dialogue dans le Don Quichotte » [...]. Mais, à part la phrase dont je vous ai parlée avec Chano, il n'y avait pas une familiarité particulière, non, non, non.

RB : Merci. Alors, Bénédicte Vauthier employait tout à l'heure l'expression de "travail de re-traduction » (et j'insiste sur le trait d'union du re-traduction) des classiques. C'est sans doute ce qui caractérise les classiques d'ailleurs, dont on peut dire qu'ils sont classiques dans la mesure où ils résistent à la traduction et exigent à intervalles réguliers d'être re-traduits. L'une des différences entre votre traduction de ces classiques donc et celle des textes précédents était que, autant vous deviez inventer pour traduire Donoso, pour traduire Sarduy ou pour traduire Goytisolo, autant vous deviez ré-inventer pour les classiques et, par conséquent, vous démarquer plus ou moins volontiers de vos prédécesseurs. Dans quelle mesure donc les traductions déjà existantes furent-elles une aide à votre travail, et dans quelle mesure purent-elles aussi constituer un obstacle ? La question se pose tout particulièrement pour Don Quichotte, où le poids de la traduction est le plus évident, avec les traductions en particulier de Louis Viardot, de Jean Cassou ou de Francis de Miomandre, pour ne citer que les plus classiques, mais $L a$ Célestine, le Buscón ou Sainte Thérèse posent en réalité tout autant ce même problème.

AS : Borges a une phrase qui est très belle et très juste en plus : « les traducteurs d'une même œuvre, les retraducteurs d'une même œuvre forment, constituent une dynastie ennemie ", c'est-à-dire qu'on traduit toujours contre ceux qui vous ont précédés. Ça c'est très clair. Alors, dans la mesure

4 N. d. E. Aline Schulman se refiere a los exámenes de la agregación francesa, concurso nacional destinado a los jóvenes que en Francia desean ejercer como profesores en la enseñanza secundaria superior. 
où on me donnait comme objectif une traduction moderne, il était évident que je n'allais pas faire ce que les autres avaient fait. Alors, deux choses que je voudrais dire. Premièrement, je n'ai pas une grosse tête, moi je ne suis pas une intello, je ne réfléchis pas tout le temps quoi, voilà. Je me suis dit «tu vas faire ce que tu sais faire, ma vieille, tu vas faire ce que tu sais faire, c'est-à-dire, tu vas traduire Cervantès comme tu traduis Goytisolo ». Et j'ai abordé les cinquante pages - vous savez, on vous demande toujours de traduire cinquante pages pour savoir si vous êtes à la hauteur. J'ai commencé à le traduire comme si je commençais à traduire un livre de Juan, sans parti pris d'archaïsme, bref, comme je le ferais d'un autre livre, voilà. Donc, ça a marché et il est évident qu'il y avait des choses qu'il fallait déplacer. Alors, ça c'est une première chose. Et alors, pas de chance [...] quand ils m'ont dit "traduisez 50 pages ", ce que je fais, je prends le livre, enfin un des deux, et puis j'ouvre et là où ça tombe je traduis ; et donc c'était tombé sur un chapitre où malheureusement, il parlait de Cide Hamete Benengeli. Alors il fallait déjà commencer à traduire ce truc. Et alors là, il faut que je vous dise quelque chose, quand même. On traduit avec sa langue bien sûr, bon c'est le français, mais on traduit aussi avec sa biographie. J'étais née au Maroc et quand vous lisez, Cide, Cide Hamete Benengeli, et je voyais Cid-, Cid-, Cide Hamete Benengeli, j’ai dit : "Mais ça ne tient pas debout leur truc ». Revenons trente secondes aux autres traducteurs, il est bien évident que je ne me suis pas gênée! Parce que pour une fois, c'était la première fois, il y avait d'autres traductions, alors, allez-y, j'en ai mis tout autour. J'avais quatre ou cinq traductions françaises, trois traductions anglaises, malheureusement ce sont les seules langues que je connais, autrement j'en aurais piochées d'autres. Et j'étais en permanence, même quand je partais en voyage, j'en amenais quelques-unes, pour être sûre de pas être toute seule. Donc, les traducteurs m'ont énormément aidée à ne pas faire ce qu'ils faisaient et parfois à faire ce qu'ils faisaient. Bon, mais revenons à ce que je disais, si je n'ai pas oublié... Qu'est-ce que je voulais dire?

RB : Sidi Hamete.

AS : Voilà. Sidi Hamete. Je me suis dit ce n'est pas possible Cide Hamete. $\mathrm{Ou}$ bien on dit le Cid et alors on se croirait chez Corneille, ou bien on dit Cide et ça ne veut rien dire. Vous savez à quoi j'ai pensé à ce moment-là ? Je me suis dit, en pensant à mon passé, à ma vie au Maroc, je me suis dit les Français auraient dit Sidi Mohammed Ben Couscous. Seulement, je ne pouvais pas aller jusque-là. Alors, je me suis dit « Benengeli » c'est « berenjena », eh comment je l'ai traduit en fait ? Vous vous souvenez vous ? Sidi Ahmed Ben Benengeli, voilà. [...] La seule chose que je pouvais me permettre... Le Ben Couscous ce n'était pas possible, pourtant c'était ça, parce que «berenjena, c'est de la nourriture », vous comprenez ? Et c'est de la nourriture que les musulmans de l'époque, les envahisseurs avaient apportée. Alors, le Ben 
Couscous ça aurait été formidable. Mais, je n'osais pas. Mais au moins je me suis coltinée avec le «Cide Hamete » et j'ai redonné, si vous voulez, ce que moi j'avais toute ma vie entendu : «Sidi Ahmed » et c'est devenu alors «Sidi Ahmed Benengeli ». Et ça dès le premier envoi que j’ai fait au Seuil, si vous voulez. J'ai été obligée de débroussailler une difficulté qui pour moi était essentielle et je l'ai fait, justement, avec ma biographie, voyez, avec ce que moi je savais du Maroc, voilà. J'ai répondu ? Oui?

RB : Oui. Et alors est-ce que pour les autres œuvres classiques que vous avez traduites, est-ce que vous avez eu ce dialogue avec des prédécesseurs aussi ? Ou est-ce que c'était moins marquant?

AS : Avec le Buscón il y en a eu un ou deux. Je regardais quand même ce qu'ils avaient fait, dans la Pléiade il y avait le Buscón qui a été traduit, alors évidemment c'était beaucoup plus classique, mais c'est vrai que quand on ne comprend pas certains passages, il est merveilleux de voir que d'autres les ont compris et bien compris, seulement ils ne les ont pas traduits peut-être comme on souhaiterait. Alors, on garde le sens, si vous voulez, et puis on change la syntaxe et le lexique. Mais ça aide énormément d'avoir un avant, si vous voulez, et pour Sainte Thérèse, c'est pareil. Je crois que ça a été la même chose sauf que je n'ai pas fait comme les autres, mais j'avais aussi les traductions précédentes de Marcelle Auclair, de comment s'appelle-t-il ce professeur de Nanterre? Qui va me souffler?

RB : Sesé ? Sesé ? [...]

AS : [...] Bernard Sesé, j'avais donc d'excellentes traductions ; et c'était très difficile de faire mieux. Voilà, donc, les traductions m'ont toujours aidée, m’ont toujours été indispensables quand elles existaient, voilà.

RB : Alors vous y avez déjà répondu partiellement, mais je voudrais revenir sur le défi que représente traduire Don Quichotte. Un auteur espagnol du XIX ${ }^{e}$ siècle, José María Sbarbi, s’était amusé à composer, en réponse à José María Asensio, un « passe-temps littéraire "sur la Intraducibilidad del Quijote, donc la non-traductibilité du Quichotte. Or, au cours d'une table ronde organisée lors des vingt-huitièmes assises de la traduction littéraire, consacrée aux « Monstres en traduction », vous affirmiez que ces monstres - alors, Don Quichotte, en l'occurrence - «ne sont pas dévorateurs », et que, bien au contraire, " ils se laissent apprivoiser ». J'aime bien cette image du traducteur qui apprivoise. Plus, même, disiez-vous, " ils ne demandent que cela, qu'on s'occupe d'eux ». Pourrait-on alors dire que traduire Cervantès, cela a été un peu pour vous répondre à une exigence qui émanait de Cervantès même dans son texte, que formulait Sanson Carrasco, à savoir que l'histoire de l'ingénieux hidalgo " soit bientôt traduite par toutes les nations et dans toutes les langues »?

AS : Écoutez, pendant que vous parlez, j'essaie de me remettre dans l'état où j'étais. Ça a été un bonheur pendant six ans. Tous les jours je savais 
que je devais faire une page et ça a été un bonheur parce que je crois que ce texte est tellement extraordinaire. Mais je rigolais toute seule en traduisant Sancho. (Je voudrais vous lire un passage.) Don Quichotte c'est, comment vous dire, il y a plusieurs tranches, il est multiple cet homme, il est la caricature du chevalier errant, mais ça c'est la première image ; après il y en a une autre, il y a le type drôlement intelligent et qui sait parfaitement ce que c'est que l'éducation et qui sait parfaitement ce que c'est que tout. (Je vais vous lire un passage.) Et puis il y a mille et une facettes, mais il y a une construction [...] Je voudrais vous dire autre chose aussi : un traducteur, ce n'est pas le traducteur seulement des mots, mais, comme le dit Mahmud Darwish : « il est le guetteur de l'ombre et de ce qu'elle révèle ». Or, dans le Quichotte, il y a plus d'ombres que je n'en ai jamais traduites dans aucun autre livre; et c'est ça qui est nourrissant et quand je dis les ombres c'est tous ces non-dits, tous ces non-dits qui changent, comment vous dire, la perception de ces ombres. Elles changent avec les époques. C'est pour ça que les traductions doivent changer. [...] Regardez! Je vous donne un exemple, je connais très bien Pierre Vidal-Naquet, mais il ne savait pas que c'est moi qui traduisais [...] notre regretté Pierre Vidal-Naquet [...]. Il me dit : «Chère Madame, j’ai lu [Don Quichotte] ; ôtez-moi d'un doute ; Cervantès était bien contre l'expulsion des Morisques? ». Je me suis dit : «zut, alors, je n'ai pas traduit pour rien »! Parce qu'avec un texte clair, qui dit les choses comme elles sont, il faut qu'on soit capable de lire l'arrière-plan de ce que veut dire Cervantès, c'est-à-dire, bien sûr qu'il est contre l'expulsion des Morisques, qu'il est contre l'éducation des femmes auxquelles on impose ceci et cela, qu'il est contre l'éducation des garçons à qui on refuse de faire de la poésie, parce que soi-disant la poésie ça ne se vend pas, etcetera, etcetera. J'ai fait une conférence qui s'appelait "Les silences de Cervantès ", c'est-à-dire, tout ce qui sort entre les rainures de ce texte, entre des ombres de ce texte et qui est la véritable substantifique moelle du Quichotte et c'est pour ça qu'on continue à le lire aujourd'hui. Et je pense que quand on le traduit de manière claire, les choses apparaissent. Alors, c'est pour ça que je me permettrai, si vous permettez, de lire juste un petit passage où tout d'un coup Don Quichotte est le grand homme, si vous voulez, il n'est plus la caricature, il n'est plus l'imbécile et c'est le prêtre qui devient l'imbécile et [...] Cervantès se permet ça.

Nous sommes au chapitre 22 de la deuxième partie. Quand Don Quichotte est donc chez le Duc et la Duchesse et puis il y a un prêtre qui lui reproche je ne sais quoi en disant « mais enfin vous êtes un imbécile » :

«Me direz-vous au moins Monsieur laquelle de mes extravagances vous autorise à me critiquer et à me condamner comme vous le faites, à m'ordonner de rentrer chez moi pour prendre soin de ma femme et de mes enfants sans même vous préoccuper de savoir s'ils existent. [Alors, je saute.] Serait-ce donc se lancer dans une vaine entreprise ou perdre son temps que de le pas- 
ser à courir les routes, en y cherchant non pas les plaisirs de ce monde mais les épreuves par lesquelles les hommes de bien atteignent à l'immortalité. Si j'étais traité de sot par des chevaliers, par des hommes d'illustre naissance, par de grands seigneurs, je le ressentirais comme une offense irréparable, mais que les pédants me considèrent comme tel, eux qui n'ont jamais fait un pas sur les sentiers de la chevalerie, je m'en moque. Chevalier je suis et chevalier je mourrai, s'il plaît à Dieu. Il y en a qui choisissent la voie spacieuse de l'ambition et de l'orgueil, d'autres celle de l'adulation servile, d'autres encore empruntent la route de l'hypocrisie trompeuse et quelques-uns le chemin de la véritable religion. Quant à moi, guidé par mon étoile, je m'aventure sur les trois sentiers de la chevalerie errante où l'on méprise l'argent, mais non l'honneur. J'ai vengé des offense, redressé des torts, châtié des insolents, vaincu des géants et abattu des monstres. Et si je suis amoureux, c'est seulement parce qu'un chevalier errant ne peut se dispenser de l'être. Mais, je reste un amant platonique et continent. Je n'ai d'autres buts que de faire le bien à tous et de ne jamais nuire à personne. À vous, Monsieur le Duc, Madame la Duchesse de juger si quiconque pense de la sorte et agit de la sorte, mérite d'être traité d'imbécile ».

Ah, c'est pas magnifique ça ? Là c'est le Quichotte. Ah, on n'est plus dans la caricature. Voilà, c'est sublime!

RB : La presse parla en 1997 , lors de la publication de votre traduction, de « la plus moderne et la plus audacieuse " des traductions en langue française du Quichotte. La difficulté, dans la traduction de Don Quichotte, comme d'ailleurs de tout classique, est qu'au transport d'une langue à l'autre s'ajoute le problème du transport d'une époque à l'autre. Et je voudrais vous demander dans ce sens quels ont été les choix les plus importants que vous aviez dû prendre ? Quelles décisions ont été particulièrement difficiles à prendre ? Quelles ont été vos trouvailles aussi ? Je pense en particulier, mais peut-être qu'il y en a d'autres, à trois choses : la traduction des archaïsmes dans le texte du Quichotte (par exemple ínsula), celle des proverbes ensuite, et puis enfin la question de la construction des phrases avec les nombreux subjonctifs posait un problème aussi, je crois, dans le rendu en français.

AS : Vaste question. C'est la traduction elle-même que vous me demandez d'expliquer. Alors, bon, je l'ai écrit dans la préface, je peux peut-être le répéter, vous savez on n'a aucune idée préconçue quand on commence. Quand on commence on ne sait pas où on va trop, trop bien. On tâte le terrain et on tâtonne, voilà. Et puis petit à petit, petit à petit, comment diraisje, il devient clair qu'on ne peut pas utiliser certains mots et qu'on peut en utiliser d'autres. Je vous donne un exemple. J'avais utilisé un proverbe dans lequel il y avait le terme... Sancho disait : "Si ça continue, si vous ne me donnez pas "je ne sais pas trop quoi" je vais tomber dans les pommes ». Et moi je me dis «ah, c'est pas mal ça ». Et puis quand j'ai relu, je me suis aperçu que ce «tomber dans les pommes » grinçait par rapport au reste. [...] À ce 
moment-là [...], j’ai acheté le dictionnaire Robert historique, et je me suis misr à chercher la date d'entrée des mots dans la langue française. Et je me suis aperçue que le «tomber dans les pommes ", c'était I8oo et quelques. Trop loin, beaucoup trop loin. Alors que tout mon effort était justement de rester aux environs de I650. Et petit à petit je me suis aperçue que - il y a des exceptions, parce qu'on ne peut pas tout faire magnifiquement bien $98 \%$, si vous voulez, des mots qui sont dans ma traduction n'entrent pas après I650 dans la langue française. Alors comment fait-on pour garder cet aspect, pour donner cet aspect moderne, c'est-à-dire pour que tout le monde comprenne? Parce que l'important, c'est qu'il faut qu'on comprenne sans notes. C'est ça qui compte. Bon, comment on va faire alors? Alors, il y a le lexique, mais le lexique en espagnol a varié beaucoup plus qu'en français, si vous voulez, des termes de 1650 ne sont plus reconnaissables aujourd'hui, alors qu'en France à partir de I650 jusqu'à aujourd'hui, on peut utiliser des termes tout à fait compréhensibles. Alors, l'important finalement, une fois qu'on a décidé ça pour le lexique, c'est la syntaxe, parce qu'il est évident que Cervantès utilise encore une syntaxe du genre latin, si vous voulez, et puis circonvolution, et puis il se répète - et je me répète -. Alors, je me demande si c'est du latin ou si c'est pour que tout le monde le comprenne, parce que c'est quand même une œuvre qui est, au départ, orale. Elle est écrite, bien sûr, mais elle est surtout lue oralement à tous ces gens qui ne savaient pas écrire, qui ne savaient pas lire, pardon. Donc, la syntaxe, donc il y a eu cet effort permanent de conserver un lexique qui était donc non pas moderne mais compréhensible par les gens d'aujourd'hui et ensuite, créer, retrouver une syntaxe qui s'accorde, comme dit Meschonnic, avec le mode de signifier d'aujourd'hui. Il ne faut pas non plus qu'il nous donne une [...] musique incompréhensible - on est dans la musique, dans la syntaxe - et si c'est une musique inaudible, le livre tombe comme pour Nathalie Sarraute. Il faut que la musique soit audible et ça, ça a été la grosse difficulté. Chaque phrase - et puis elles sont longues ces phrases -, chaque phrase a été une difficulté. Il fallait s'accrocher, il fallait s'accrocher ! J'étais en train de lire en venant l'interview d'Erri de Luca dans Le monde des livres. C'est un homme qui adore la montagne et qui vit de la montagne et moi j'ai fait beaucoup de montagne et pendant que je [...] travaillais le Quichotte, quand j'étais en train de traduire justement cette deuxième partie, j'ai fait mes 4000 et c'est ça traduire finalement. Traduire un livre aussi difficile, c'est commencer en sachant qu'on va y arriver même si c'est difficile et on y arrive, on y arrive. C'est dur, mais on y arrive.

RB : Oui, oui, très bien. Merci. Alors, vous mentionnez justement à l'instant la deuxième partie et on sait donc que le Don Quichotte est une œuvre qui n'est pas intrinsèquement homogène : c'est un roman qui se compose de deux parties, publiées à dix ans de distance, en I605 et en I6I5, deux parties 
qui diffèrent entre elles non seulement par certains aspects de la construction narrative, mais aussi même par leur langue, leur style. Et je voudrais savoir si vous avez pu prendre en compte cette évolution à l'œuvre dans le roman, qui le travaille en quelque sorte de l'intérieur et comment ça a pu affecter votre traduction?

AS : Ben, j'étais complètement idiote. J'ai commencé à traduire la deuxième partie. Alors, évidemment, qui va se mettre à commencer « en un lugar de la Mancha ", c'est la dernière chose qu'on traduit. Donc, je ne pouvais pas commencer comme ça. J'ai commencé par la deuxième partie et ce qui a été extrêmement difficile après, c'est qu'il a fallu que je redescende, si vous voulez, il a fallu que je m'habitue à un auteur de quinze ans plus jeune, dix ans plus jeune, c'est-à-dire qui écrivait de manière tout à fait différente, et un auteur qui [...] n'avait pas encore idée, premièrement, de son succès et, deuxièmement, du fait qu'il y aurait un autre bonhomme qui viendrait lui piquer le morceau. Alors, j'ai fait une bêtise. Si c'était à refaire, je ne le referais pas. Mais bon, ça ne sera jamais un point pour moi en tout cas. Mais ça a été extrêmement difficile de passer du style du deuxième au style du premier, qui est tout de même un autre auteur qui écrit. Il est non seulement plus jeune, mais il n'a pas cette perspective encore. D'abord parce qu'il n'y a pas eu l'expulsion des Morisques, bon elle se prépare, mais elle n'a pas eu lieu, voyez-vous, et cela ça a bouleversé la deuxième partie.

RB : Alors, au cours de la même table ronde des assises de la traduction littéraire que j'ai évoquée tout à l'heure, vous mentionniez aussi, à propos de votre travail de traductrice, un « journal de bord», dans lequel vous consigniez un ensemble de considérations relatives à votre travail. Alors je voudrais vous demander quel rôle ce journal de bord a joué dans votre travail ? Comment ça se passait concrètement ? En quoi cette écriture en marges de la traduction éclaire cette traduction, en quoi elle peut aider aussi ? Et se poserait aussi la question de savoir si d'autres journaux ont existé, de ce genre, en marge d'autres traductions?

AS : Vous parlez de mes traductions?

RB : De vos traductions, évidemment.

AS: Bon alors, je vous dis tout de suite qu'il y a des passages intéressants, voilà, et puis d'autres qui ne valent rien. C'est vrai qu'il y a des moments où on a besoin, comme on dit en espagnol, de « desahogarse ». Comment on dit ça en français, «desahogarse ».

RB : Se soulager?

AS : Se soulager, voilà. Se soulager, mais on ne peut se soulager avec personne, parce que personne n'est dans votre truc et ce n'est pas Annie Morvan que j'allais embêter pour lui dire «tu sais ça et ça et ça ». Alors, je me soulageais sur mon journal et j'écris la plupart du temps : « Je n'en peux plus. C'est trop long ». Voyez, des choses comme ça et « et pourtant il faut 
tenir » et puis d'autres choses aussi mais bon, c'est trop long, je ne pourrais pas vous en parler maintenant. Mais je suis en train d'écrire un livre sur la traduction et la vie et il y aura des passages de mon journal dans ce livre, donc je préfère ne pas trop en parler pour l'instant. Voilà, mais c'est vrai qu'on a besoin d'une fenêtre ouverte de temps en temps, de respirer, de crier, de soupirer, de cracher, voilà.

RB : Merci. Alors, votre traduction de Don Quichotte a eu une vie, je crois, après sa publication. En particulier, je pense au fait que, en 2006, je crois, vous avez monté un spectacle à partir du roman, Quichotte, joué à Cannes, à Nanterre, et à Belfort, je crois. Je voudrais vous demander dans quel rapport ce spectacle, qui suppose une réécriture du texte, se situe face à la traduction ? Est-ce que ce spectacle exprime, par exemple, un désir d'appropriation du texte de Cervantès - on en parlait tout à l'heure déjà, de l'appropriation du texte par le traducteur -, un texte qui serait devenu si familier par le fait même d'avoir été traduit, et, si tel est le cas, dans quel sens cette appropriation tend?

AS : Alors, je vais corriger un petit peu ce que vous dites, je crois. Ça n'est pas une réécriture du tout, c'est un redécoupage et moi je n'ai fait que dire amen, corriger des petites choses, mais dire amen à ce que le metteur en scène, qui est Didier Galas, faisait. Donc, on n'a absolument pas réécrit le Quichotte, simplement on l'a découpé et de manière remarquable. Pour moi, ça a été un spectacle. Vous savez, le sens du Quichotte, il est multiple, mais ce garçon a réussi à mettre en scène le Quichotte comme personne, parce qu'il y a eu au moins une quinzaine de mises en scène du Quichotte après lui, et en vingt ans, personne ne l'a fait aussi bien que lui. Alors, c'étaient des séquences, exactement le genre, la manière dont Cervantès divise son texte, enfin, ce sont des histoires bout à bout et ce sont des chapitres, vous savez, " el cuento de nunca acabar », c'est ça. Et il en a fait justement des séquences extrêmement visuelles et extrêmement belles sans changer absolument rien au texte, mais en mettant le texte en scène. Alors, évidemment qu'il y a des paroles, mais c'est surtout la mise en scène qui était belle. Donc il n'y a pas eu de réécriture, dans aucune des représentations du Quichotte qui ont pu se faire. En dernier c'était le théâtre de Bagnolet qui était très bien. Il y en a eu d'autres qui étaient moins bonnes. Enfin, il y en a eu beaucoup, beaucoup, beaucoup. Mais jamais ils n'ont touché au texte. Ils ont simplement redécoupé le texte, mais jamais touché au texte [...] j'étais là pour refuser ça.

$\mathrm{RB}$ : Toujours le respect.

AS : Voilà le respect.

RB : Maintenant, si vous le permettez, évoquons les textes classiques dont la traduction a suivi celle de Don Quichotte. Il vient d'être question de théâtre, et le texte qui vint, après Don Quichotte, ce fut La Célestine, dans le registre des textes classiques. Comment se fit ce choix? 
AS : Alors, autant jusqu'au Quichotte, je n'avais moi jamais osé dire, je voudrais traduire ceci ou cela puisque je n'existais pas. Je ne pouvais pas avoir des désirs, bon, et bien là, enfin, après le Quichotte, j’avais le droit de dire « ah, je voudrais traduire ceci ». Et comme chez Fayard il y avait cet être exceptionnel qui était Claude Durand, avec qui j'avais un excellent rapport... Il était directeur, il avait été directeur au Seuil à l'époque où je traduisais déjà et puis bon et je lui dis «écoutez, je voudrais traduire $L a C e ́$ lestine ». Il m'a dit "mais comment donc, chère amie ", voyez, à l'époque on vous acceptait ces choses-là. Donc, j'ai traduit encore cinquante pages et tout s'est bien passé. Et pourquoi La Célestine? Alors, si vous voulez, étant étudiante, j'avais donc eu La Célestine au programme et j'avais eu Santa Teresa; et je m'étais toujours dit «Santa Teresa je la traduirai ». J'avais 20 ans et je m'étais dit "Santa Teresa je la traduirai », mais comme c'est la personne la plus redoutable à traduire, j'ai fini avec elle, voyez. Voilà, mais en tout cas, La Célestine c'est parce que je l'avais beaucoup travaillée, parce qu'on en parlait énormément avec Juan, il adorait ça, on en parlait souvent et Juan était vraiment, comment dirais-je, un autre, un autre moi-même, c'est ridicule de dire ça, mais je veux dire, on était en confiance, même dans la parole, en dehors des livres. Et si Juan me parlait beaucoup de ça, je me suis dit «allez, on y va ", voilà.

RB : Alors, justement dans le texte de Goytisolo que vous avez traduit et placé en guise de préface au début de votre traduction de La Célestine, Juan Goytisolo caractérise la langue de La Célestine dans des termes qui donnent une idée de la difficulté de ce qu'il y avait à traduire, et de la nouveauté que ce langage-là supposait dans la langue espagnole aussi, dans le Xv et le XVI siècle. Alors, je me permets de le citer : « Rojas joue à la perfection des différents registres d'un langage inédit, brutal, libéré des conventions qu'on lui imposait jusqu'alors : il frôle l'obscénité, se vautre dans la rudesse, accélère vertigineusement le rythme, enfile les phrases comme on enfile des perles, les malmène, transforme la matière verbale pour en faire un organisme prodigieusement vivant. » Du coup, la question que je voudrais vous poser c'est quelles furent, pour vous et face à ce texte, les difficultés majeures au moment de le traduire?

AS : Premièrement, j'ai un peu oublié, mais enfin là n'est pas la question. C'était très difficile aussi. Mais, comment vous dire, je me suis atenue, atenido, je me suis tenue aux mêmes critères que ceux du Quichotte, c'est-à-dire, un lexique qui soit non pas moderne, donc sans notes toujours, mais qui ne soit, comment dirais-je, qui ne soit pas postérieur à I650 et puis une syntaxe qui elle aussi soit fluide. Alors, je crois que l'essentiel c'était de découvrir qu'au fond si Rojas, parce qu'il le fait, fait parler les serviteurs comme les maîtres, parce qu'il n'y a pas vraiment de grande différence entre la manière dont parle Calixto et ou Sempronio o Pármeno, ¿no ? Et ce que ça veut dire 
quelque part, c'est qu'ils ne valent pas mieux les uns que les autres, c'est-àdire que Calixte et, j'ai oublié son nom, Madame...

RB : Mélibée

AS : Mélibée, que Calixte et Mélibée ne valent pas mieux que leurs servantes et serviteurs respectifs et c'est ça que j'ai découvert moi en lisant, voyez, en lisant attentivement, en traduisant, c'est ça que j'ai découvert. [...] Partout c'est le stupre et le lucre et ils sont tous au même stade, si vous voulez, voilà. Alors, la Célestine n'est rien, n'est pas moins que Calixte, n'est pas moins que Mélibée, bref, et j'ai essayé, si vous voulez, de traduire tout le monde au même niveau. Voilà, c'est ce que j'ai essayé de faire pour donner une cohérence à ce texte et une facilité de lecture aussi ah, voilà.

RB : Alors, vous avez pu dire que « sans ce travail sur des textes d'écrivains vivants, présents, espèce de duel face à face, [vous] n'auriez jamais eu le courage de [vous] lancer dans la traduction du Quichotte ». Dès lors, se pose la question de savoir, mais on en a un peu parlé déjà, la question de savoir quel fut le rôle, dans vos traductions du Quichotte mais aussi des autres classiques, des auteurs récents que vous aviez traduits? Le travail sur Goytisolo, en particulier, vous avait-il préparée pour porter un certain regard sur les classiques espagnols, et ce qu'ils supposent pour l'« hispanité »? Plus concrètement, dans votre traduction de La Célestine, pour revenir à elle, apparaissent deux textes en guise de préface : le premier de Goytisolo, traduit par vous-même, le second, de Carlos Fuentes, traduit par Céline Zins. Que signifie la présence de ces deux auteurs, que vous avez bien connus, au seuil de votre traduction de La Célestine?

AS : Pour faire vendre ! En gros c'est ça, mais il y a autre chose aussi derrière. Non, en fait, c'est juste, parce que... Ce que je dois dire c'est que, tout de même, il faut rendre hommage à l'université. Nous sommes dans l'université, enfin, dans l'École la plus prestigieuse de France et qui est connue dans le monde entier et il faut vraiment « rendre à César »... L'université nous a quand même, m'a quand même enseigné beaucoup de choses. Elle m'a enseigné la traduction au sens où on n'a pas le droit de dire n'importe quoi, ah, le respect du texte, moi c'est en faisant de la version du grec que j'ai appris le respect du texte, et ça c'est très très important et il faut le dire. Il faut dire que l'université aide énormément à faire du bon boulot après. Bon, donc, d'un point de vue de la version; et puis c'est à l'université que j'ai étudié Sainte Thérèse et que j'ai commencé à adorer Sainte Thérèse, c'est à l'université que j'ai étudié Don Quichotte, donc je connaissais quand même quand on a passé l'agreg' avec Don Quichotte, on sait quand même un peu. J'ai passé l'agreg avec La Célestine, on sait quand même un peu. Donc, tout ça, ça gargouillait à l'intérieur de moi, si vous voulez. Et c'était quelque chose

5 N.d.É. Aline Schulman se refiere a la "agregación", concurso nacional, abreviada en "agreg". 
qui est sorti. Tout d'un coup je me suis dit « Mais au fond ce qu'il faut c'est traduire les grands textes ». C'était un besoin. C'est ridicule parce que c'est dur, c'est dur. Vous vous rendez compte : le Buscón! Mais il le fallait. Je ne sais pas pourquoi. Je me suis dit, si je sais, je me suis dit «pour les étudiants, ils ne vont pas passer par où moi je suis passée ", parce que, franchement, la traduction de La Célestine de Heugas, c'était pire que le texte. Alors, ce n'est pas possible et le Buscón il y en avait une de, comment il s'appelle, c'était du XVIII', XVII ${ }^{e}$, comment il s'appelait ce Monsieur, alors c'était bourré de contre-sens. Voilà, alors il y a eu ça aussi. Je me suis dit « puisque maintenant je peux, je crois que j'ai les forces, eh ben je vais leur donner ». Ce n'était pas tellement de l'altruisme, c'était aussi du bonheur pour moi, mais il y avait de ça, voilà.

$\mathrm{RB}$ : Je crois qu'ils vous sont reconnaissants aussi. Alors, justement vous parliez du Buscón, un autre monument, un autre monstre qu'il fallait apprivoiser, et dont la langue est, je crois, tout particulièrement difficile aussi, car plus ingénieuse que ne l'est celle de Cervantès. La question qui se pose serait de savoir si pour le Buscón - alors vous disiez que pour La Célestine vous avez en gros appliqué les mêmes recettes que pour Don Quichotte -, est-ce que le même procédé pouvait fonctionner pour le Buscón?

AS : Alors, pour le Buscón, c'est tout à fait autre chose. D’abord c'est un gars qui a vingt et quelques années, il est tout jeune. Vous me direz que ça, Rojas aussi était jeune, mais, c'est, comment vous dire, le Buscón, quand on le lit bien, enfin, quand on le traduit en particulier, c'est une BD. C'est une bande dessinée où Quevedo reprend des tas de choses qu'on dit, qu'on écrit, des jokes, des jeux de mots, voyez, ce sont des jeux de mots - [...] les histoires d'aubergine [etcetera] - mais il reprend des jeux de mots qui étaient à la mode à l'époque et puis il les enrichit d'une certaine manière. Alors, [...] heureusement il y a plein de notes [...], ils nous disent que ceci vient de cela, que c'était quelque chose que l'on disait, etcetera. Et je l'ai traduit comme une BD. Vous me direz: «Qu'est-ce que ça veut dire?». Je ne sais pas vous le dire moi-même, mais dans l'idée que c'était lu comme une BD, c'est-à-dire des personnages que l'on reconnait [...], des jokes, c'est-à-dire des chistes que l'on reconnaît aussi, qui ne sont pas neufs, c'est ça qui est intéressant. Ce qui n'est pas neuf non plus, c'est son antisémitisme, son antiarabisme. Alors j'ai essayé là de louvoyer parce qu'il y avait des trucs qui m'étaient vraiment extrêmement difficiles à traduire, parce que je n'en voulais pas. Alors je me suis vengée par ailleurs. Je n'ai pas raconté ça... je peux vous le dire en deux minutes : il avait dit en particulier des juifs que c'étaient ces gens qui ont de grands nez, mais qui ne leur servent même pas à sentir le cochon. Alors, je trouvais que ce n'était évidemment pas gentil. Alors, à un moment donné où un des voleurs qui est avec le Buscón en prison, on ne l'aime pas, je ne sais pas pourquoi, et on lui dit : «oh, mais toi, tu vas faire de la fumée, tú vas a hacer humo ». Ça veut dire qu'il va finir sur le bucher. Et bien moi j'ai traduit 
en pensant au four crématoire, voyez comme quoi on peut se venger, même quand on est traducteur. "Tu vas partir en fumée », comme on dit que tous les gens partaient en fumée, vous avez entendu l'expression ? [...] voilà je me suis vengée en traduisant comme cela. Ceux qui s'en rendront compte, s'en rendront compte, mais moi je le sais. Voilà.

RB : Nous n'étions pas dans le registre de la complicité ou de la cordialité avec l'auteur? Il y a un rapport plus tendu.

AS : Non, il n'est pas sympa du tout, pas du tout, pas du tout, pas du tout. Il est très adroit, extrêmement adroit, mais c'est un type que je n'aurais pas aimé rencontrer.

RB : Mais que vous avez souhaité traduire?

AS : Oui, parce que c'était encore une fois un livre qui est de una gracia, je ne sais pas comment on dit ça en français, c'était comique, c'était le comique de l'époque, et il est bon qu'on reconnaisse comment est fait le comique de l'époque, en fait, c'est les tartes à la crème de l'époque, si vous voulez. Voilà, je crois que j'ai dit ce que je voulais dire.

RB : Merci. Alors vous évoquez au début, tout au début de l'entretien l'importance qu'avait eue Saint Jean de la Croix dans les cours que vous aviez reçus. Donc, passez maintenant de Saint Jean de la Croix à Thérèse d'Avila. C'est le dernier texte dont je voudrais parler. Traduire Les Chemins de la perfection, pour citer le titre que vous avez donné à votre choix de textes, a été un exercice inédit, je crois, auquel ni Donoso, ni Sarduy, ni Goytisolo, ni Cervantès, ni Quevedo ne vous avaient préparée. Vous avez pu dire, à propos du texte de Cervantès, que " on ne fait pas ce qu'on veut avec un texte. C'est lui qui nous impose son rythme. » Et au cours d'un entretien donné, je crois, à Radio France Internationale en 20I5, au moment où vous prépariez le Sainte Thérèse, vous affirmiez que la traduction de Thérèse d'Avila vous avait changée, chose qu'aucune des traductions antérieures n'avait faite. Respect absolu du texte, modestie et humilité face au texte, sont les enseignements de la mystique du Carmel, dont le style s'efforce pourtant de tendre vers l'absolue clarté. Humilité devant une clarté. Pourriez-vous s'il vous plaît évoquer simplement pour nous ce qui vous incita à traduire Thérèse d'Avila, et ce que supposa cette expérience?

AS : Alors, merci de me poser la question parce que vraiment avec Thérèse, parce que moi je l'appelle Thérèse, ça s'est passé de manière tout à fait différente, si je compare avec les traducteurs précédents. Thérèse, d'abord, c'est une mystique, c'est une femme inouïe, je veux dire, il suffit d'entrer dans El Libro de la vida pour se rendre compte à qui on a à faire. Alors, Cervantès on ne se rend pas tellement compte à qui on a affaire. On a un livre qui est magnifique, mais, comment vous dire, Thérèse c'est un personnage et on sait combien elle a souffert. Le problème a été le suivant : c'est que moi, je suis une athée absolue et que j'avais affaire à une femme qui était totalement en Dieu. 
Mais, je suis partie comme ça sur les chapeaux de roue en me disant «allez, va, je t'aurai bien toi aussi ». Et ce qui est arrivé, c'est quelque chose d'incroyable, c'est que j'ai été obligée d'arrêter. Quand elle a commencé à nous faire le traité d'oraison, c'est-à-dire à partir du chapitre I2 du Livre de la vie. Au départ, je ne voulais traduire que Le Livre de la vie, voyez-vous, et j'y étais partie comme ça. Et quand elle cesse de parler de sa propre vie et qu'elle commence à entrer vraiment dans la théorie de l'oraison, je me suis rendu compte que je n'arrivais plus. Je commençais à traduire le matin et puis j'allais voir ce qui se passait ailleurs, puis je revenais 30 secondes et je ressortais. Je ne pouvais plus rester à ma table. Alors, à ce moment-là, c'était en mars 20I2, on m'a demandé de faire un topos sur Santa Teresa au Mans. Et j'ai arrêté à ce moment-là de traduire et j'ai écrit ce truc-là et j'ai dit « écoutez, je ne peux pas, voilà, bon, je ne peux pas ». C'est un texte qui est tout le temps sous l'œil de la censure, donc il n'y a rien de ludique dans ce texte. Or, le traducteur, s'il veut créer un tant soit peu dans la traduction, il faut qu'il y ait un aspect ludique, il faut qu'il y ait un aspect ouvert. Or, chez Thérèse, rien. Pourquoi ? Parce qu'elle est sous l'œil de la censure en permanence, de l'Inquisition. Alors, à ce moment-là, j'ai terminé mon texte en disant : «Voilà, j'ai arrêté parce que je ne pouvais plus, je n'en pouvais plus, je n'en voulais plus ». Et du fond de l'amphi, il y a une voix, féminine évidemment, c'est les femmes qui savent, qui connaissent, qui me dit : «Madame, et si c'était elle qui ne voulait pas de vous?». Alors, je l'ai regardée, tout le monde a éclaté de rire, mais moi ça a ouvert, si vous voulez, une vérité en moi. Mais évidemment, je ne peux pas traduire des textes purement spirituels. Je n'ai pas le droit de les traduire, parce que moi je ne suis pas assez spirituelle. À ce moment-là, qu'est-ce que j'ai fait ? Je me suis dit, enfin avec l'aide des copains qui m'ont aidée parce que j'étais plutôt down, down, down... Ils m'ont dit : « Mais fais une anthologie des textes que toi tu peux traduire, qu'elle te permet, elle, de traduire ». Et c'était ça, le chemin de la perfection. C'est une anthologie des textes qu'elle m'a permis de traduire. Voyez l'histoire avec Sainte Thérèse, ce que c'était. Incroyable ! Mais tout est vrai, point par point de tout ce que je vous ai dit. Et à partir de là, j'ai fait mon anthologie et je me suis remis à traduire et j'ai fini. Voilà.

RB : C'est une belle réussite. [...] Et je vous remercie pour votre témoignage sur l'ensemble de votre travail de traduction et nous vous remercions donc collectivement pour votre présence aujourd'hui ici. Merci beaucoup.

AS : C’est moi qui vous remercie de m'avoir invitée ce soir, vraiment. 